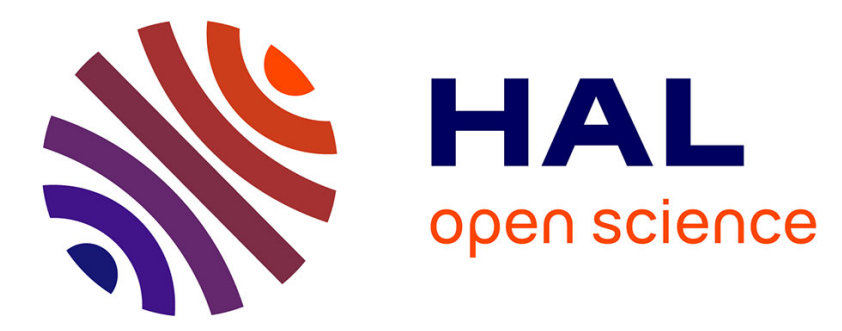

\title{
Predictive strain-gradient homogenization of a pantographic material with compliant junctions
}

Baptiste Durand, Arthur Lebée, Pierre Seppecher, Karam Sab

\section{To cite this version:}

Baptiste Durand, Arthur Lebée, Pierre Seppecher, Karam Sab. Predictive strain-gradient homogenization of a pantographic material with compliant junctions. Journal of the Mechanics and Physics of Solids, inPress, 10.1016/j.jmps.2021.104773 . hal-03529670

\section{HAL Id: hal-03529670 \\ https://hal-enpc.archives-ouvertes.fr/hal-03529670}

Submitted on 17 Jan 2022

HAL is a multi-disciplinary open access archive for the deposit and dissemination of scientific research documents, whether they are published or not. The documents may come from teaching and research institutions in France or abroad, or from public or private research centers.
L'archive ouverte pluridisciplinaire HAL, est destinée au dépôt et à la diffusion de documents scientifiques de niveau recherche, publiés ou non, émanant des établissements d'enseignement et de recherche français ou étrangers, des laboratoires publics ou privés. 


\title{
Predictive strain-gradient homogenization of a pantographic material with compliant junctions
}

\author{
Baptiste Durand $^{\mathrm{a}}$, Arthur Lebée ${ }^{\mathrm{a}}$, Pierre Seppecher ${ }^{\mathrm{b}}$, Karam Sab $^{\mathrm{a}}$ \\ ${ }^{a}$ Laboratoire Navier (UMR 8205), École des Ponts ParisTech, Université Gustave Eiffel, CNRS, Marne-la-Vallée, France \\ ${ }^{b}$ Institut de Mathématiques de Toulon, Université de Toulon, Toulon, France
}

\begin{abstract}
This paper presents an architectured material featuring significant strain-gradient effects and called pantographic material. It is a plate made of a single and continuous linear elastic material containing voids and thus is easy to fabricate. The pattern consists of triangles connected by thin junctions and arranged in such a way that two floppy strain modes are present. A homogenization scheme based on the two-scale asymptotic expansion is suggested, keeping only significant strain-gradient contributions in the homogenized energy by means of an adequate projection. The predictions from the homogenization scheme are validated against a full-scale simulation and yield very good $L^{2}$ error estimates whereas the classical first-gradient homogenization fails. Furthermore, the relative position of the unit-cell of the full-scale computation does not have a significant influence on the quality of the prediction.
\end{abstract}

Keywords: Strain-gradient continuum, Higher-order homogenization, Metamaterial, Compliant mechanism

\section{Introduction}

Architectured materials are heterogeneous materials with a designed microstructure, most often periodic. Their macroscale behavior emerges rather from the microstructure than from the properties of the constituent materials. Recent advances in manufacturing techniques, such as additive manufacturing processes, raised a considerable interest in these materials as they provide solutions to the demand for better mechanical performances in engineering applications. Indeed, even in elasto-statics, a theoretical result shows that a vast variety of macroscale mechanical behaviors may be obtained from an arbitrary mixture of materials (Camar-Eddine and Seppecher, 2003): not only the classical first-gradient elasticity theory but also generalized continua (Cosserat and Cosserat, 1909; Mindlin, 1964) or metamaterials (Kadic et al., 2019). Hence, a new framework has emerged, called material by design, which consists in designing the microstructure of an architectured material with design tools such as topology optimization, in order to obtain the desired behavior at the macroscopic scale. This framework relies deeply on predictive up-scaling methods relating the microscopic properties of the architectured material to its overall mechanical behaviour. In these methods, the microstructure may be modelled either as a discrete mechanical system or as a continuous medium. However, following 
a material by design framework, we focus here on media which are continuous at the microscopic scale with reasonable volume fraction and are thus easy to fabricate and can result from topology optimization.

Up-scaling methods for a continuous medium leading to first-gradient elasticity are now well established in statics as well as in dynamics. They are based on the leading order of the two-scale asymptotic expansion and convergence results have been proved (Jikov et al., 1994). Hence architectured material properties have been widely explored and optimized in this framework ((Bendsøe and Sigmund, 2004; Amstutz et al., 2010; Wang et al., 2014; Zhu et al., 2017; Thomsen et al., 2018) among many others). This is not the case of up-scaling methods for generalized continua which were derived either from discrete microstructures or still require ad hoc hypothesis or specific stiffness contrast assumptions for a continuous microstructure.

In the present paper we focus on the linear strain-gradient elasticity theory which, unlike firstgradient theory, can take into account fast variations of the strain fields. In dynamics, this is mandatory when the wavelength $\lambda$ becomes very small compared to the overall size of the material $T$. In such a case, boundary layers may be neglected and strain-gradient elasticity brings out some dispersive properties of the architectured material as illustrated for instance in (Rosi and Auffray, 2016). The natural framework for investigating wave propagation in architectured materials is Bloch wave decomposition (Lee and Yang, 1973; Willis, 1980; Auriault and Bonnet, 1985; Nassar et al., 2015; Vondřejc et al., 2017). Its close link with the two-scale asymptotic expansion (with respect to the small parameter $\eta=t / T$ characteristic of the scale separation) was pointed out and a strain-gradient up-scaling method capturing acoustic branches of the dispersion diagram was suggested as well as higher-order convergence justified when $\eta$ tends to zero. (Santosa and Symes, 1991; Allaire et al., 2016). Hence, topology optimization in this framework has already been performed (Bonnet and Cornaggia, 2017; Allaire and Yamada, 2018; Bonnet et al., 2018; Cornaggia and Bellis, 2020).

The case of elasto-statics investigated in this paper is more challenging. Indeed, without a wavelength to compare with, size effects in statics must be compared to the overall size $T$ of the elastic body and are meant to vanish when $T$ tends to infinity. Hence, strain-gradient effects are classically presented as higher-order corrections with a small typical characteristic or internal length. In this direction, numerous up-scaling methods leading to an effective strain-gradient continuum were suggested. Following historical contributions from Mindlin (1964) and Germain (1973) a family of approaches is based on quadratic periodic boundary conditions applied to the unit-cell (Gologanu et al., 1997; Kouznetsova et al., 2002; Auffray et al., 2010). These methods are based on the intuition that the unitcell may be curved when strain gradients are significant. However, they present inconsistencies which were reviewed by Monchiet et al. (2020) who pointed out a relation with the second family of up-scaling methods based on higher orders of the two-scale asymptotic expansion. Indeed, as for dynamics, higherorder contributions terms involve higher gradients of the macroscopic strain (Bakhvalov and Panasenko, 
1989; Gambin and Kröner, 1989; Triantafyllidis and Bardenhagen, 1996; Boutin, 1996). A consistent variational derivation of higher-gradient theories was suggested by Smyshlyaev and Cherednichenko (2000) and a higher-order convergence result was obtained with very specific assumptions on the boundary conditions and the averaging method. This result has not been extended to more general boundary conditions and strain-gradient effects seems to remain very limited for the investigated microstructures (Peerlings and Fleck, 2001; Ameen et al., 2018).

This motivated another direction for designing microstructures presenting significant effective strain-gradient effects. Indeed, when the first-gradient effective constitutive law is degenerate, there exists some zero-energy, or floppy, deformation modes with a non-vanishing strain. In this case, strain-gradient elastic energy may become preponderant. This requires to break out of the classical assumptions in homogenization methods which always lead to a non-degenerate strain energy. To that aim, two approaches were followed. First, mechanisms, that is discrete microstructures with perfect junctions, were considered: with a well-chosen configuration of perfect, or zero-energy, junctions, it is possible to design microstructures with floppy strain modes and derive a strain-gradient homogenized model (Alibert et al., 2003; Dell'Isola et al., 2016). However, this requires an educated guess of the microstructure which is also rather difficult to fabricate (Dell'Isola et al., 2019). Second, one has to assume specific contrast and geometry for the continuous microstructure (Pideri and Seppecher, 1997; Abdoul-Anziz and Seppecher, 2018a,b; Abdoul-Anziz et al., 2021). Again, this leads to a rigorous justification of strain-gradient continua in elasto-statics which was validated in (Jakabčin and Seppecher, 2020) but is specific to each microstructure and not suitable for topology optimization.

The main objective of this paper is to assess the prediction of the higher-order effective behavior given by the two-scale asymptotic expansion applied to a continuous microstructure featuring significant strain-gradient effects. To that aim, we consider the pantographic microstructure from Abdoul-Anziz and Seppecher (2018a) simply made of triangles connected by compliant junctions (Figure 1). Indeed, slender bars investigated in (Jakabčin and Seppecher, 2020) are strongly subject to buckling at the microscopic level and thus are not suitable for an experimental study nor to practical use. On the contrary, it is expected that considering solid triangles will mitigate this non-linear effect. However, no higher-order convergence result is established for this class of microstructure, motivating the present study.

First, we investigate in Section 2 the behavior of the architectured material we consider when connections between triangles become thin and show that first-gradient elasticity is not able to correctly predict the homogenized behavior. Then, in Section 3, the higher-order homogenization scheme based on the asymptotic expansion is recalled. As the contrast is here infinite and the shape of the microstructure is evolving with thinner and thinner junctions, the considered structure falls out of the classical assumptions in homogenization. The strain-gradient homogenized stiffness tensor given by the higher-order homogenization corresponds to different intrinsic lengths. When the strain does not 
belong to a degenerate direction of the first-gradient homogenized stiffness tensor, these lengths are extremely small (of the order of the size of the periodic cell) and, at the macroscopic level, the effect of such strain-gradient terms can be ignored. On the contrary, this effect becomes important, maybe preponderant, when the strain belongs to a degenerate direction of the first-gradient homogenized stiffness tensor. Hence we project the strain-gradient homogenized stiffness tensor in these directions (Section 4). This presents two advantages. First the model that we derive is actually a macroscopic one as it does not try to describe the equilibrium solution at the scale of the unit-cell. Secondly the model leads to well-posed problems, which is not always the case when the whole strain-gradient tensor is retained (Allaire et al., 2016; Smyshlyaev and Cherednichenko, 2000). Finally, in Section 5, we perform extensive numerical simulations in order to compare the strain-gradient model and the first-gradient model to a numerical reference solution. The simulations reveal the very good predictions of the strain-gradient model.

\section{The continuous pantographic metamaterial}

\subsection{The microstructure}

The proposed pantographic metamaterial is a bi-dimensional body with a periodic microstructure. This microstructure is composed of identical triangles and of rhombi connected at their vertices by thin junctions, as shown in Figure 1a. It is made of a homogeneous isotropic linear elastic material. We denote $\boldsymbol{c}$ the stiffness tensor of this constituent material. The remaining space is empty.
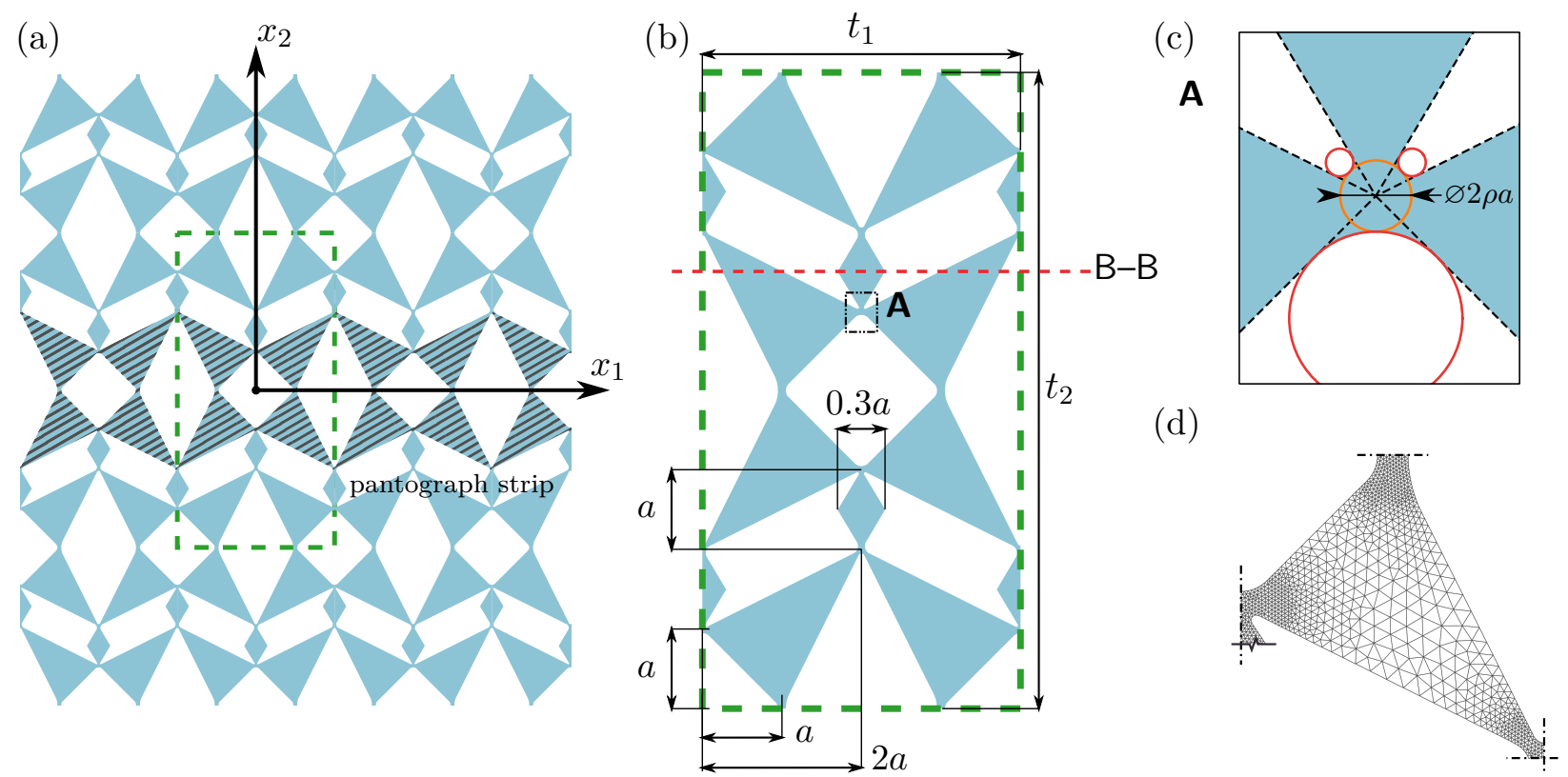

Figure 1: (a) The periodic microstructure (b) Geometric configuration of the unit-cell (c) Geometric construction of the junctions (d) Illustration of mesh density around the junctions

The chosen unit-cell used for the computation of the effective behavior is rectangular and its geometry is presented in Figure 1b. The geometry of the microstructure depends on two parameters: 
the length $a$ and the dimensionless junction thinness $\rho$. The dimensions of the unit-cell in the global coordinate system are $t=t_{1}=4 a$ and $t_{2}=8 a$. Hence, $t$ is the typical size of the unit-cell and $\tau=t_{2} / t_{1}=2$ is the aspect ratio of the unit-cell. The area fraction of solid with respect to the unit-cell area $t_{1} t_{2}$ is denoted $\phi$.

The junction geometry is defined as follows: a circle of radius $\rho a$ is centered at the common vertex of adjacent triangles and rhombi (orange circle in Figure 1c); then each void angle sector is smoothed with a circle (drawn in red in Figure 1c) tangent to both edges and the orange circle. The parameter $\rho$, representative of the junction thinness has a significant influence on the behavior of the metamaterial. Indeed, the shape of the junctions makes them much more compliant to bending moment than to traction or shear forces. It is thus expected that they almost behave like revolute joints when $\rho$ is small enough.

More precisely, the unit-cell configuration allows macroscopic strains with low energy called floppy modes. First, the arrangement of triangles as horizontal strips in Direction 1 allows almost free stretch in this direction. This mode is denoted pantograph mode in reference to the classical mechanism. One pantograph strip is hatched on Figure 1a. Secondly, rhombi which connect vertically pantograph strips allow an almost free horizontal relative motion in Direction 1 between all strips. These floppy modes were already investigated for structures containing perfect junctions (Alibert et al., 2003) and $\Gamma$-convergence results were obtained when the unit-cell is the assembly of slender beams (Anziz, 2018; Abdoul-Anziz and Seppecher, 2018b).

\subsection{Emergence of strain-gradient effects}

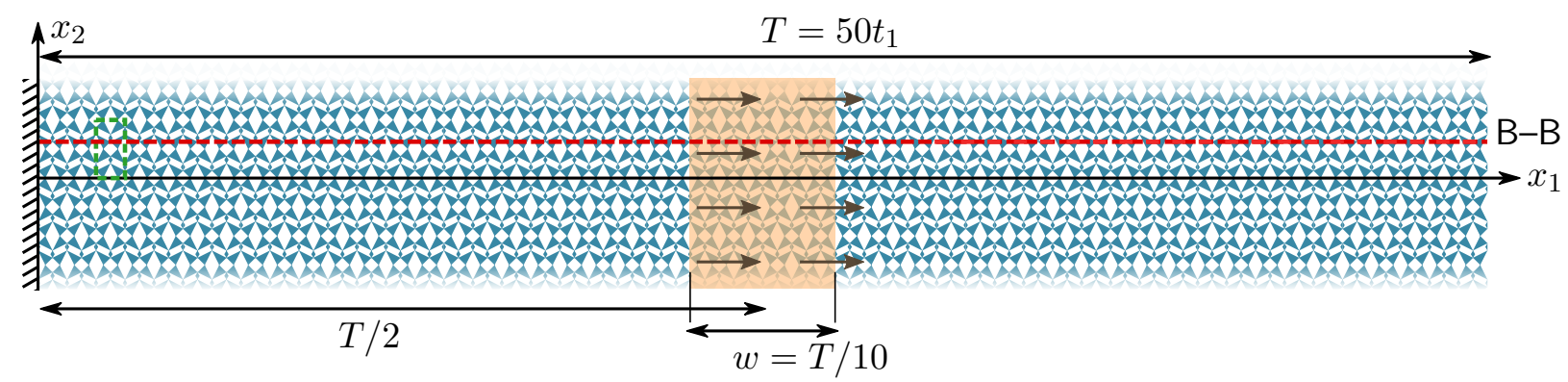

Figure 2: 2D linear elastic boundary value problem for the pantographic metamaterial. The domain is periodic in Direction 2.

We consider a 2D rectangular body that is clamped at $x_{1}=0$ and free at $x_{1}=T$ in plane-strain linear elasticity (Figure 2). In Direction 2, periodic boundary conditions are applied along the lateral boundaries. The sample is made of the pantographic metamaterial, with 50 unit-cells in the length $T$. It is subjected to a load $\underline{f}$ uniformly distributed on a central strip of the elastic body. More precisely $\underline{\boldsymbol{f}}=\underline{\boldsymbol{e}}_{1}$ for $\frac{T}{2}-\frac{w}{2}<x_{1}<\frac{T}{2}+\frac{w}{2}$, with $w=\frac{T}{10}$.

Our reference displacement solution $\underline{\boldsymbol{u}}^{\text {fs }}$ is obtained using a full-scale numerical simulation, i.e. using a standard FEM formulation and a mesh that represent the whole microstructure accurately. 


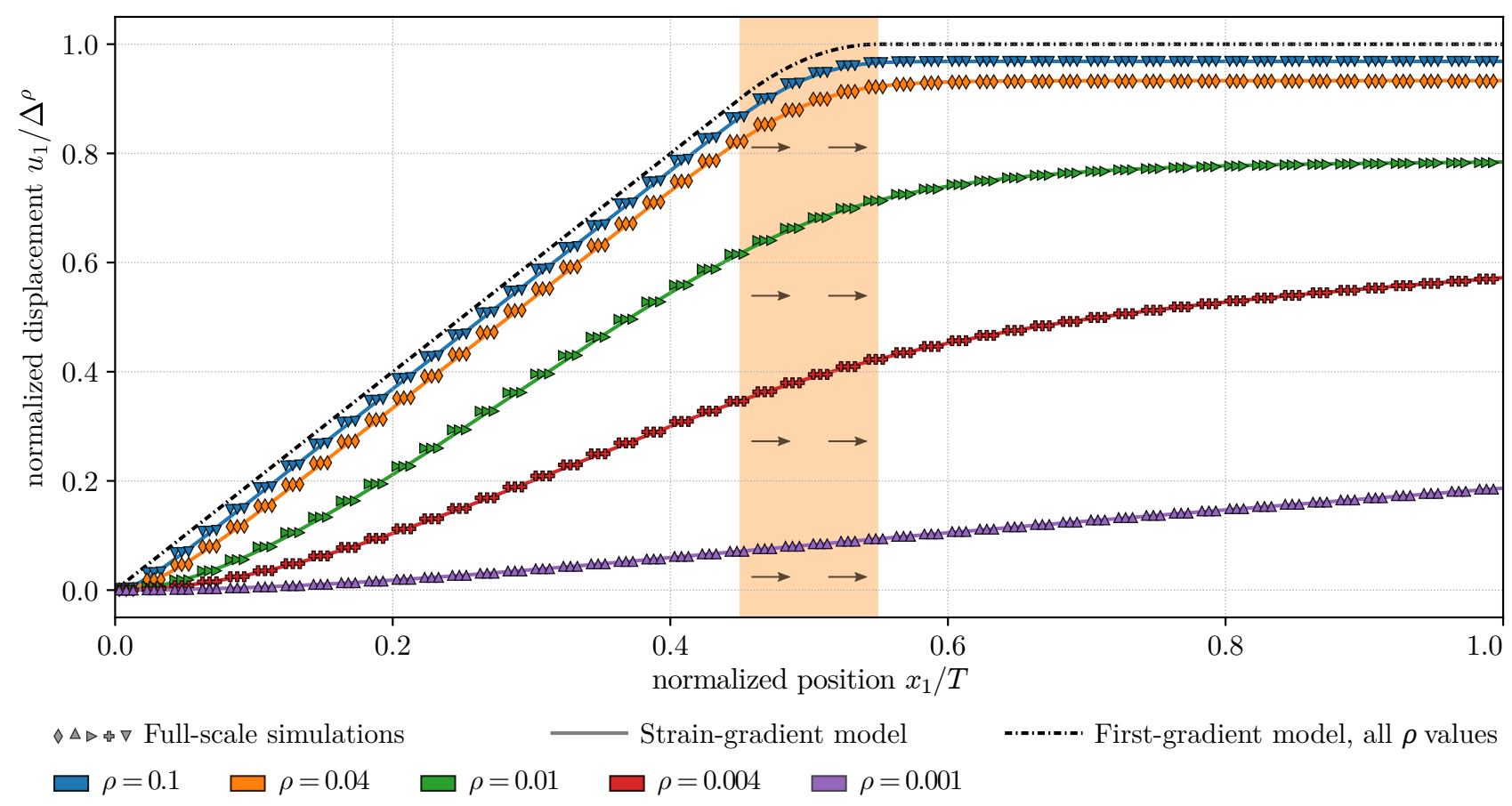

Figure 3: Distribution of the longitudinal displacement $u_{1}$ along the B-B line (Figures $1 \mathrm{~b}$ and 2) for several $\rho$ values. For each full-scale simulation (markers), the first-gradient (dashed line) and strain-gradient (solid lines) homogenized solutions are also plotted.

Full details of the finite-element simulations are given in Section 5.2. They are performed for a series of values of the junction thinness $\rho$ in the interval $\left[10^{-3}, 10^{-1}\right]$.

In order to check whether a standard homogenized model based on a first-gradient continuum is able to describe this equilibrium from the macroscopic point of view, we derive, in a closed form, the solution of the corresponding boundary value problem. The standard homogenized stiffness tensor $\boldsymbol{C}^{\rho}$ will be computed in Section 3. Owing to symmetries, the homogenized displacement in Direction 2 $\left(U_{2}\right)$ vanishes and the horizontal displacement $U_{1}$ is independent of $x_{2}$. It can be expressed as follows:

$$
U_{1}\left(x_{1}\right)= \begin{cases}\Delta^{\rho} \frac{2 x_{1}}{T} & \text { for } 0 \leq x_{1} \leq \frac{T-w}{2} \\ \Delta^{\rho}\left(1-\frac{1}{T w}\left(x_{1}-\frac{T+w}{2}\right)^{2}\right) & \text { for } \frac{T-w}{2} \leq x_{1} \leq \frac{T+w}{2} \\ \Delta^{\rho} & \text { for } \frac{T+w}{2} \leq x_{1} \leq T\end{cases}
$$

where $\Delta^{\rho}=\frac{\phi f w L}{2 C_{111}^{\rho}}$ is the displacement on the right side of the plate $\left(x_{1} \geq \frac{T+w}{2}\right)$ predicted by the first-gradient homogenized model. Note that, when the junction thinness $\rho$ decreases, the homogenized stiffness $C_{1111}^{\rho}$ decreases and consequently the value of $\Delta^{\rho}$ increases. Anyway, the function $U_{1} / \Delta^{\rho}$ is independent of it and consequently of $\rho$.

Figure 3 shows the profile along a horizontal line of $U_{1} / \Delta^{\rho}$ and compares it to the horizontal displacement $u_{1} / \Delta^{\rho}$ obtained by the full-scale simulation and normalized with the homogenized first-gradient displacement $\Delta^{\rho}$ computed for each value of $\rho$.

It appears that the first-gradient homogenized model leads to an overestimation of the horizontal displacement $u_{1}^{\mathrm{fs}}$. The discrepancy between the homogenized displacement $U_{1}$ and the full-scale 
displacement $u_{1}^{\mathrm{fs}}$ is visible even for rather thick junctions and becomes very significant when $\rho \rightarrow 0$. It also appears that this discrepancy cannot be corrected by simply correcting the value of $C_{1111}^{\rho}$ given by standard homogenization theory. Indeed the profiles obtained by the full-scale simulation are not constant in the free part $\left(x_{1} \geq \frac{T+w}{2}\right)$ of the plate and are thus fundamentally different from the profile given by (1).

The reason for the failure of the classical periodic homogenization when junctions are getting thinner is the following: the first-gradient homogenized stiffness tensor $\boldsymbol{C}^{\rho}$ becomes ill-conditioned as $C_{1111}^{\rho} \rightarrow 0$ when $\rho \rightarrow 0$. Hence, higher-order effects, related to components of the strain gradient become significant. Whereas classical homogenization predicts larger and larger displacement $\Delta^{\rho}$, the actual displacement remains limited because the clamped boundary condition prevents the relative motion of the triangles inside the microstructure.

Ahead of our development, we also plot in Figure 3 the macroscale displacement that we will obtain using the equivalent homogeneous strain-gradient medium derived in Section 4. It appears clearly that it describes in a very accurate way the equilibrium of the considered architectured material from the macroscopic point of view.

\section{Higher-order homogenization from the two-scale asymptotic expansion}

In the present section, the formal two-scale asymptotic expansion procedure is briefly recalled for a microstructure made of a homogeneous linear elastic material and voids. The geometry of the unit-cell is fixed. The expansion is carried out up to the second order and the derivation of a strain-gradient homogenized energy is recalled.

\subsection{The two-scale asymptotic expansion}

The periodic medium is defined on a $2 \mathrm{D}$ connected domain of typical size $T$ obtained by the repetition of a unit-cell of typical size $t$. The repetitive unit-cell is constituted of a linear elastic material and voids. Let us work in a dimensionless framework by introducing the space variable $\underline{\boldsymbol{Y}}=\underline{\boldsymbol{x}} / T$ and the relative size of the unit-cell $\eta=t / T$. We also divide the displacement field by $T$ in order to work with a dimensionless field $\underline{\boldsymbol{u}}$. We assume that the displacement is prescribed on the external boundary $\partial \Omega^{\text {ext }}$, whereas the boundary $\partial \Omega^{\text {int }}$ of the voids is traction-free. The equilibrium displacement under the action of a body force $\underline{f}$ concentrated on the solid part of the domain satisfies the boundary value problem

$$
\left\{\begin{array}{l}
\underline{\operatorname{div}}(\boldsymbol{\sigma})+\underline{\boldsymbol{f}}=0, \quad \boldsymbol{\sigma}=\boldsymbol{c}: \varepsilon^{\eta} \quad \text { and } \quad \boldsymbol{\varepsilon}=\boldsymbol{\nabla}^{s} \underline{\boldsymbol{u}} \text { on } \Omega \\
\underline{\boldsymbol{u}}=\underline{\mathbf{0}} \text { on } \partial \Omega^{\mathrm{ext}} \quad \text { and } \quad \boldsymbol{\sigma} \cdot \underline{\boldsymbol{n}}=\underline{\mathbf{0}} \text { on } \partial \Omega^{\mathrm{int}}
\end{array}\right.
$$

where $\underline{\text { div }}$ stands for the divergence operator, $\boldsymbol{\nabla}^{s}$ is the symmetric part of the gradient operator and $\underline{\boldsymbol{n}}$ denotes the normal to the boundary $\partial \Omega^{\text {int }}$ of the voids. 
Notations. All developments are made in a fixed Cartesian frame endowed with an orthonormal basis $\left(\underline{e}_{1}, \underline{e}_{2}\right)$. Hence, it is useful to introduce the following tensorial notations. Vectors $\underline{X}$ are underlined and higher-order tensors $\boldsymbol{X}$ are simply in boldface. When dealing with components, Greek indices $\alpha, \beta, \gamma_{.}=1,2$ are used and Einstein's summation convention is followed for repeated indices. The transpose operation $\boldsymbol{X} \mapsto \boldsymbol{X}^{\top}$ is applied to any order tensors $\boldsymbol{X}$ by reversing the indices order as follows: $\left(\boldsymbol{X}^{\boldsymbol{\top}}\right)_{\alpha \beta \ldots \psi \omega}=X_{\omega \psi \ldots \beta \alpha}$. Four symbols are defined: $(\cdot),(:),(\vdots)$ and (:) for the contraction products on, respectively, one, two, three and four indices. By convention, closest indices are successively summed together in contraction products. For instance, contracting three times the six-order tensor $\boldsymbol{X}$ with the third-order tensor $\boldsymbol{Y}$ writes as: $(\boldsymbol{X}: \boldsymbol{Y})_{\alpha \beta \gamma}=\left(X_{\alpha \beta \gamma \delta \lambda \mu} Y_{\mu \lambda \delta}\right)$. Finally, Kronecker symbol is denoted $\delta_{\alpha \beta}\left(\delta_{\alpha \beta}=1\right.$ if $\alpha=\beta, \delta_{\alpha \beta}=0$ otherwise).

Double-scale representation. As we intend to study the behavior of the structure in the homogenized limit, that is when $\eta$ becomes smaller and smaller, we parametrize Problem (2) and all associated quantities by $\eta$. Moreover we search for a double-scale representation of these quantities of the form $\underline{\boldsymbol{u}}^{\eta}(\underline{\boldsymbol{Y}}, \underline{\boldsymbol{y}}), \boldsymbol{\sigma}^{\eta}(\underline{\boldsymbol{Y}}, \underline{\boldsymbol{y}}), \varepsilon^{\eta}(\underline{\boldsymbol{Y}}, \underline{\boldsymbol{y}})$ where $\underline{\boldsymbol{y}}$ is the microscopic space variable defined by

$$
y_{1}=\frac{x_{1}}{t_{1}}-\left\lfloor\frac{x_{1}}{t_{1}}\right\rfloor=\frac{Y_{1}}{\eta}-\left\lfloor\frac{Y_{1}}{\eta}\right\rfloor \quad \text { and } \quad y_{2}=\frac{\underline{x}_{2}}{t_{1}}-\frac{t_{2}}{t_{1}}\left\lfloor\frac{x_{2}}{t_{2}}\right\rfloor=\frac{Y_{2}}{\eta}-\tau\left\lfloor\frac{Y_{2}}{\tau \eta}\right\rfloor .
$$

Here $\lfloor x\rfloor$ stands for the floor of $x$. Note that $\underline{\boldsymbol{y}}$ belongs to the rescaled cell $[0,1] \times[0,2]$, more precisely to the solid part $\mathcal{Y}$ of it.

The body load $\underline{\boldsymbol{f}}$, concentrated on the solid part of the unit-cell, is assumed to vary slowly. Hence its double-scale representation is assumed to be of the form $\frac{1}{\phi} \underline{\boldsymbol{F}}(\underline{\boldsymbol{Y}})$. The normalization with the volume fraction of solid $\phi=\frac{|\mathcal{Y}|}{\tau}$ ensures that $\underline{\boldsymbol{F}}$ will correspond to the body load per unit surface of the macroscopic domain $\Omega$.

The scale-separation assumption consists in assuming the independence of the two coordinate variables $\underline{\boldsymbol{Y}}$ and $\underline{\boldsymbol{y}}$. It enables us to rewrite the spatial derivation operator $\underline{\boldsymbol{\nabla}}_{x}$ under the form

$$
\underline{\nabla}=\underline{\nabla}_{Y}+\frac{1}{\eta} \underline{\nabla}_{y}
$$

Expansion. Treating $\eta$ as a perturbation parameter, functions that represent mechanical quantities are written as formal power series of $\eta$ :

$$
\underline{\boldsymbol{u}}^{\eta}(\underline{\boldsymbol{Y}}, \underline{\boldsymbol{y}})=\sum_{k=0}^{\infty} \eta^{k} \underline{\boldsymbol{u}}^{k}(\underline{\boldsymbol{Y}}, \underline{\boldsymbol{y}}), \quad \boldsymbol{\varepsilon}^{\eta}(\underline{\boldsymbol{Y}}, \underline{\boldsymbol{y}})=\sum_{k=-1}^{\infty} \eta^{k} \varepsilon^{k}(\underline{\boldsymbol{Y}}, \underline{\boldsymbol{y}}), \quad \boldsymbol{\sigma}^{\eta}(\underline{\boldsymbol{Y}}, \underline{\boldsymbol{y}})=\sum_{k=-1}^{\infty} \eta^{k} \boldsymbol{\sigma}^{k}(\underline{\boldsymbol{Y}}, \underline{\boldsymbol{y}})
$$

Both these expressions as series and the expanded derivation operator (4) are incorporated in the equations of Problem (2). Then, each equation of this system is broken down into a set of equations where each one corresponds to the terms in $\eta^{p}$ for a given power $p$. These elementary equations are then gathered in order to form a sequence of auxiliary boundary value problems defined on the solid part $\mathcal{Y}$ of the rescaled unit-cell. For each problem of order $p$, the main unknown is the displacement field $\underline{\boldsymbol{u}}^{p}$ while the field $\underline{\boldsymbol{u}}^{p-1}$ is considered as an input of the problem. The problems are nested and solved successively. 


\subsection{Resolution of the first auxiliary problems and resulting asymptotic series}

Leading order displacement. The case of the order $p=0$ yields:

$$
\begin{cases}\underline{\boldsymbol{\nabla}}_{y}^{s} \underline{\boldsymbol{u}}^{0}=0 & \text { on } \mathcal{Y}, \\ \underline{\boldsymbol{u}}^{0}(\underline{\boldsymbol{Y}}, \underline{\boldsymbol{y}}) & \text { periodic with respect to } \underline{\boldsymbol{y}} .\end{cases}
$$

The structure $\Omega$ that we consider is connected. Hence the displacement $\underline{\boldsymbol{u}}^{0}$ is a rigid-body displacement. Owing to the periodicity condition, it is a global translation of the unit-cell:

$$
\underline{\boldsymbol{u}}^{0}(\underline{\boldsymbol{Y}}, \underline{\boldsymbol{y}})=\underline{\boldsymbol{U}}^{0}(\underline{\boldsymbol{Y}})
$$

which cannot be determined at this step.

First-order auxiliary problem. The auxiliary problem of order $p=1$ is a boundary value problem for the displacement $\underline{\boldsymbol{u}}^{1}$ :

$$
\begin{cases}\underline{\operatorname{div}}_{y} \boldsymbol{\sigma}^{0}=\underline{\mathbf{0}} & \text { on } \mathcal{Y}, \\ \boldsymbol{\sigma}^{0}=c: \varepsilon^{0} & \text { on } \mathcal{Y}, \\ \varepsilon^{0}=\boldsymbol{E}^{0}+\underline{\boldsymbol{\nabla}}_{y}^{s} \underline{\boldsymbol{u}}^{1} & \text { on } \mathcal{Y}, \\ \boldsymbol{\sigma}^{0} \cdot \underline{\boldsymbol{n}}=\underline{\mathbf{0}} & \text { on } \partial \mathcal{Y}, \\ \underline{\boldsymbol{u}}^{1}(\underline{\boldsymbol{Y}}, \underline{\boldsymbol{y}}) & \text { periodic with respect to } \underline{\boldsymbol{y}},\end{cases}
$$

where the macroscopic strain $\boldsymbol{E}^{0}(\underline{\boldsymbol{Y}})$ is defined as:

$$
\boldsymbol{E}^{0}=\underline{\nabla}_{Y}^{s} \underline{U}^{0}
$$

The solution $\underline{\boldsymbol{u}}^{1}$ of this linear boundary value problem is again defined up to an undetermined macroscopic translation, now denoted $\underline{\boldsymbol{U}}^{1}$. This solution depends linearly on the source term $\boldsymbol{E}^{0}$ and thus can be expressed as the linear superposition $\underline{\boldsymbol{u}}^{1}(\underline{\boldsymbol{Y}}, \underline{\boldsymbol{y}})=\boldsymbol{h}^{1}(\underline{\boldsymbol{y}}): \boldsymbol{E}^{0}(\underline{\boldsymbol{Y}})+\underline{\boldsymbol{U}}^{1}(\underline{\boldsymbol{Y}})$ or, in terms of indices,

$$
u_{\alpha}^{1}(\underline{\boldsymbol{Y}}, \underline{\boldsymbol{y}})=h_{\alpha \beta \gamma}^{1}(\underline{\boldsymbol{y}}) E_{\gamma \beta}^{0}(\underline{\boldsymbol{Y}})+U_{\alpha}^{1}(\underline{\boldsymbol{Y}}) .
$$

The third-order displacement localization tensor $\boldsymbol{h}^{1}$ that relates $\underline{\boldsymbol{u}}^{1}$ to $\boldsymbol{E}^{0}$ is uniquely defined once its mean value is fixed by ${ }^{1}$

$$
\left\langle\boldsymbol{h}^{1}\right\rangle=0
$$

and can be determined by solving elementary elasticity problems on $\mathcal{Y}$. Clearly the associated strain also depends linearly on $\boldsymbol{E}^{0}$ and we have

$$
\varepsilon^{0}(\underline{\boldsymbol{Y}}, \underline{\boldsymbol{y}})=\boldsymbol{a}^{1}(\underline{\boldsymbol{y}}): \boldsymbol{E}^{0}(\underline{\boldsymbol{Y}})
$$

\footnotetext{
${ }^{1}$ The averaging operator is defined as $\langle f\rangle=\frac{1}{\tau} \int_{\mathcal{Y}} f(\underline{\boldsymbol{y}}) \mathrm{d} \underline{\boldsymbol{y}}$. Note that $\langle 1\rangle=\phi$.
} 
where the strain localization tensor $\boldsymbol{a}^{1}$ is the fourth-order tensor defined by

$$
a_{\alpha \beta \gamma \delta}^{1}=I_{\alpha \beta \gamma \delta}^{\mathrm{sym}}+\frac{1}{2}\left(\partial_{y_{\beta}} h_{\alpha \gamma \delta}^{1}+\partial_{y_{\alpha}} h_{\beta \gamma \delta}^{1}\right) \quad \text { with } \quad I_{\alpha \beta \gamma \delta}^{\mathrm{sym}}=\frac{1}{2}\left(\delta_{\alpha \gamma} \delta_{\beta \delta}+\delta_{\alpha \delta} \delta_{\beta \gamma}\right) .
$$

Finally we notice that the stress tensor is given by

$$
\sigma^{0}(\underline{\boldsymbol{Y}}, \underline{y})=c:\left(\boldsymbol{a}^{1}(\underline{y}): \boldsymbol{E}^{0}(\underline{\boldsymbol{Y}})\right)=\left(\boldsymbol{c}: \boldsymbol{a}^{1}(\underline{\boldsymbol{y}})\right): \boldsymbol{E}^{0}(\underline{\boldsymbol{Y}}) .
$$

Second-order auxiliary problem. The auxiliary problem of order $p=2$ reads

$$
\begin{cases}\underline{\operatorname{div}}_{y} \boldsymbol{\sigma}^{1}+\underline{\operatorname{div}}_{Y} \boldsymbol{\sigma}^{0}+\frac{1}{\phi} \underline{\boldsymbol{F}}=\underline{\mathbf{0}} & \text { on } \mathcal{Y}, \\ \boldsymbol{\sigma}^{1}=\boldsymbol{c}: \boldsymbol{\varepsilon}^{1} & \text { on } \mathcal{Y}, \\ \boldsymbol{\varepsilon}^{1}=\underline{\boldsymbol{\nabla}}_{Y}^{s} \underline{\boldsymbol{u}}^{1}+\underline{\boldsymbol{\nabla}}_{y}^{s} \underline{\boldsymbol{u}}^{2} & \text { on } \mathcal{Y}, \\ \boldsymbol{\sigma}^{1} \cdot \underline{\boldsymbol{n}}=\underline{\mathbf{0}} & \text { on } \partial \mathcal{Y}, \\ \underline{\boldsymbol{u}}^{2}(\underline{\boldsymbol{Y}}, \underline{\boldsymbol{y}}) & \text { periodic with respect to } \underline{\boldsymbol{y}} .\end{cases}
$$

We remark first that periodicity conditions imply that $\left\langle\underline{\mathbf{d i v}}_{y} \boldsymbol{\sigma}^{1}\right\rangle=\underline{\mathbf{0}}$. Hence the mean value of the first equation reads

$$
\underline{\operatorname{div}}_{Y} \Sigma^{0}+\underline{\boldsymbol{F}}=\underline{\mathbf{0}} \quad \text { with } \quad \boldsymbol{\Sigma}^{0}=\left\langle\boldsymbol{\sigma}^{0}\right\rangle .
$$

From equation (14), we deduce

$$
\boldsymbol{\Sigma}^{0}=\boldsymbol{C}: \boldsymbol{E}^{0} \quad \text { with } \quad \boldsymbol{C}=\left\langle\boldsymbol{c}: \boldsymbol{a}^{1}\right\rangle
$$

Recalling that $\boldsymbol{E}^{0}=\underline{\nabla}_{Y}^{s} \underline{\boldsymbol{U}}^{0}$, and using Dirichlet boundary conditions, we recover the classical firstorder homogenized problem. This is a well-posed elasticity problem where the first-order homogenized constitutive equation (17) results from the determination of the first-order strain localization tensor $\boldsymbol{a}^{1}$ which has been determined in the previous step. The homogenization procedure usually ends there. Let us however study more deeply the second-order auxiliary problem which, taking into account equations (16), (17) and (10), can be written

$$
\begin{cases}\underline{\operatorname{div}}_{y} \boldsymbol{\sigma}^{1}+\left(\boldsymbol{c}: \boldsymbol{a}^{1}-\frac{1}{\phi} \boldsymbol{C}\right): \boldsymbol{K}^{0}=\underline{\mathbf{0}} & \text { on } \mathcal{Y}, \\ \boldsymbol{\sigma}^{1}=\boldsymbol{c}: \boldsymbol{\varepsilon}^{1} & \text { on } \mathcal{Y}, \\ \boldsymbol{\varepsilon}^{1}=\boldsymbol{p}^{1}(\underline{\boldsymbol{y}}): \boldsymbol{K}^{0}+\boldsymbol{E}^{1}+\underline{\boldsymbol{\nabla}}_{y}^{s} \underline{\boldsymbol{u}}^{2} & \text { on } \mathcal{Y}, \\ \boldsymbol{\sigma}^{1} \cdot \underline{\boldsymbol{n}}=\underline{\mathbf{0}} & \text { on } \partial \mathcal{Y}, \\ \underline{\boldsymbol{u}}^{2}(\underline{\boldsymbol{Y}}, \underline{\boldsymbol{y}}) & \text { periodic with respect to } \underline{\boldsymbol{y}},\end{cases}
$$

where $\boldsymbol{E}^{1}=\underline{\nabla}_{Y}^{s} \underline{U}^{1}$ and $\boldsymbol{K}^{0}$ stand respectively for the macroscopic strain associated to the displacement field $\underline{\boldsymbol{U}}^{1}$ and strain gradient associated to the displacement field $\underline{\boldsymbol{U}}^{0}: K_{\alpha \beta \gamma}^{0}(\underline{\boldsymbol{Y}})=\partial_{Y_{\gamma}} E_{\alpha \beta}^{0}(\underline{\boldsymbol{Y}})$. The fifth-order tensor $\boldsymbol{p}^{1}$ is defined by

$$
p_{\alpha \beta \gamma \delta \epsilon}^{1}(\underline{\boldsymbol{y}})=\frac{1}{2}\left(\delta_{\beta \gamma} h_{\alpha \delta \epsilon}^{1}(\underline{\boldsymbol{y}})+\delta_{\alpha \gamma} h_{\beta \delta \epsilon}^{1}(\underline{\boldsymbol{y}})\right) .
$$


The solution $\underline{\boldsymbol{u}}^{2}$ of Problem (18) is again determined up to a global translation, now denoted $\underline{\boldsymbol{U}}^{2}(\underline{\boldsymbol{Y}})$. It depends linearly of its source terms $\boldsymbol{K}^{0}$ and $\boldsymbol{E}^{1}$. Again, solving elementary problems uniquely determines a localization tensor $\boldsymbol{h}^{2}(\underline{\boldsymbol{y}})$ satisfying $\left\langle\boldsymbol{h}^{2}\right\rangle=0$ and such that

$$
\underline{\boldsymbol{u}}^{2}(\underline{\boldsymbol{Y}}, \underline{\boldsymbol{y}})=\boldsymbol{h}^{2}(\underline{\boldsymbol{y}}): \boldsymbol{K}^{0}(\underline{\boldsymbol{Y}})+\boldsymbol{h}^{1}(\underline{\boldsymbol{y}}): \boldsymbol{E}^{1}(\underline{\boldsymbol{Y}})+\underline{\boldsymbol{U}}^{2}(\underline{\boldsymbol{Y}})
$$

We then deduce the existence of an associated strain localization tensor $\boldsymbol{a}^{2}$ such that

$$
\varepsilon^{1}(\underline{\boldsymbol{Y}}, \underline{\boldsymbol{y}})=\boldsymbol{a}^{2}(\underline{\boldsymbol{y}}): \boldsymbol{K}^{0}(\underline{\boldsymbol{Y}})+\boldsymbol{a}^{1}(\underline{\boldsymbol{y}}): \boldsymbol{E}^{1}(\underline{\boldsymbol{Y}})
$$

The formal series. The induction may be pursued up to any higher order. Each auxiliary problem yielding a higher-order macroscopic displacement $\underline{\boldsymbol{U}}^{p}$ and new localization fields $\boldsymbol{h}^{p}$ and $\boldsymbol{a}^{p}$ related to higher gradients of the macroscopic displacements $\underline{\boldsymbol{U}}^{0}$. Following Boutin (1996), Smyshlyaev and Cherednichenko (2000) and others, it is convenient to introduce the series of the macroscopic displacement:

$$
\underline{\boldsymbol{U}}(\underline{\boldsymbol{Y}})=\sum_{p=0}^{\infty} \eta^{p} \underline{\boldsymbol{U}}^{p}(\underline{\boldsymbol{Y}})
$$

as well as the corresponding strain $\boldsymbol{E}$ and strain-gradient $\boldsymbol{K}$. This allows to rewrite formally the expansion of the displacement (5) as:

$$
\underline{\boldsymbol{u}}^{\eta}(\underline{\boldsymbol{Y}}, \underline{\boldsymbol{y}}) \simeq \underline{\boldsymbol{U}}(\underline{\boldsymbol{Y}})+\eta \boldsymbol{h}^{1}(\underline{\boldsymbol{y}}): \boldsymbol{E}(\underline{\boldsymbol{Y}})+\eta^{2} \boldsymbol{h}^{2}(\underline{\boldsymbol{y}}): \boldsymbol{K}(\underline{\boldsymbol{Y}})+\ldots
$$

and the expansion of the strain as:

$$
\varepsilon^{\eta}(\underline{\boldsymbol{Y}}, \underline{\boldsymbol{y}}) \simeq \boldsymbol{a}^{1}(\underline{\boldsymbol{y}}): \boldsymbol{E}(\underline{\boldsymbol{Y}})+\eta \boldsymbol{a}^{2}(\underline{\boldsymbol{y}}): \boldsymbol{K}(\underline{\boldsymbol{Y}})+\ldots
$$

\subsection{Deriving equivalent continua from the two-scale asymptotic expansions}

In this second part of the homogenization scheme, we focus on approximations of the macroscopic energy density. The idea is to truncate the series (23) and to compute the corresponding energy. Our aim is the second-order truncation but we first recall the results obtained for shorter truncations.

Zeroth order truncation. The truncation of $\underline{\boldsymbol{u}}^{\eta}$ at order $p=0$ corresponds to the approximation $\underline{\boldsymbol{u}}^{\eta}(\underline{\boldsymbol{Y}}, \underline{\boldsymbol{y}}) \simeq \underline{\boldsymbol{U}}(\underline{\boldsymbol{Y}})$. Using the expanded derivation operator (4), one easily gets that the associated strain is $\varepsilon^{\eta} \simeq \boldsymbol{E}(\underline{\boldsymbol{Y}})$ and thus uniform on the unit-cell. The corresponding strain energy average on the unit-cell corresponds to the Voigt approximation of the homogenized strain energy:

$$
\left\langle\frac{1}{2} \varepsilon^{\eta}: c: \varepsilon^{\eta}\right\rangle \simeq \frac{1}{2} \boldsymbol{E}: \phi \boldsymbol{c}: \boldsymbol{E}
$$

which is known to be a very rough approximation. The reason appears clearly when comparing approximation $\varepsilon^{\eta} \simeq \boldsymbol{E}(\underline{\boldsymbol{Y}})$ with expansion (24) : the error due to this approximation is of order $\eta^{0}$. 
First-order truncation. The truncation of $\underline{\boldsymbol{u}}^{\eta}$ at order $p=1$ corresponds to the approximation

$$
\underline{\boldsymbol{u}}^{\eta}(\underline{\boldsymbol{Y}}, \underline{\boldsymbol{y}}) \simeq \underline{\boldsymbol{U}}(\underline{\boldsymbol{Y}})+\eta \boldsymbol{h}^{1}(\underline{\boldsymbol{y}}): \boldsymbol{E}(\underline{\boldsymbol{Y}})
$$

The related strain field is obtained by applying the expanded derivation operator (4) and taking into account Definitions (13) and (19). We get

$$
\varepsilon^{\eta}(\underline{\boldsymbol{y}}, \underline{\boldsymbol{Y}}) \simeq \boldsymbol{a}^{1}(\underline{\boldsymbol{y}}): \boldsymbol{E}(\underline{\boldsymbol{Y}})+\eta \boldsymbol{p}^{1}(\underline{\boldsymbol{y}}): \boldsymbol{K}(\underline{\boldsymbol{Y}})
$$

From now on, for the sake of simplicity, we assume that the unit-cell is centrosymmetric. Indeed, this assumption implies that odd tensors like $\left\langle\boldsymbol{a}^{\boldsymbol{1}^{\top}}: \boldsymbol{c}: \boldsymbol{p}^{1}\right\rangle=0$ must vanish. Computing the strain energy average on the unit-cell leads then to:

$$
\left\langle\frac{1}{2} \varepsilon^{\eta}: c: \varepsilon^{\eta}\right\rangle \simeq \frac{1}{2}\left[\boldsymbol{E}: \boldsymbol{C}: \boldsymbol{E}+\eta^{2} \boldsymbol{K}^{\top}: \boldsymbol{D}^{\sharp}: \boldsymbol{K}\right],
$$

where $\boldsymbol{C}=\left\langle\boldsymbol{a}^{1^{\top}}: \boldsymbol{c}: \boldsymbol{a}^{1}\right\rangle$ is the first-order effective tensor already ${ }^{2}$ introduced in (17) and $\boldsymbol{D}^{\sharp}$ is the sixth-order tensor defined as:

$$
\boldsymbol{D}^{\sharp}=\left\langle\boldsymbol{p}^{1^{\top}}: \boldsymbol{c}: \boldsymbol{p}^{1}\right\rangle .
$$

The macroscopic strain energy density (28) corresponds to a strain-gradient model. However, comparing (27) to expansion (24) shows that the error in this approximation of the strain is of order $\eta$ and thus that the approximation of the energy density we obtain is not reliable at order $\eta^{2}$. It can only be confidently used at order $\eta^{0}$ where it reads $\frac{1}{2} \boldsymbol{E}: \boldsymbol{C}: \boldsymbol{E}$ and corresponds to effective energy associated to the classical homogenized problem (16), (17).

Second-order truncation. At the order $p=2$, the approximation of the displacement field is

$$
\underline{\boldsymbol{u}}^{\eta}(\underline{\boldsymbol{Y}}, \underline{\boldsymbol{y}}) \simeq \underline{\boldsymbol{U}}(\underline{\boldsymbol{Y}})+\eta \boldsymbol{h}^{1}(\underline{\boldsymbol{y}}): \boldsymbol{E}(\underline{\boldsymbol{Y}})+\eta^{2} \boldsymbol{h}^{2}(\underline{\boldsymbol{y}}): \boldsymbol{K}(\underline{\boldsymbol{Y}})
$$

from which we deduce the related strain field

$$
\varepsilon^{\eta}(\underline{\boldsymbol{y}}, \underline{\boldsymbol{Y}}) \simeq \boldsymbol{a}^{1}(\underline{\boldsymbol{y}}): \boldsymbol{E}(\underline{\boldsymbol{Y}})+\eta \boldsymbol{a}^{2}(\underline{\boldsymbol{y}}): \boldsymbol{K}(\underline{\boldsymbol{Y}})+\eta^{2} \boldsymbol{p}^{2}(\underline{\boldsymbol{y}}):\left(\underline{\boldsymbol{\nabla}}_{Y} \boldsymbol{K}(\underline{\boldsymbol{Y}})\right)
$$

where $\boldsymbol{p}^{2}$ is the sixth order tensor defined by

$$
p_{\alpha \beta \gamma \delta \epsilon \zeta}^{2}=\frac{1}{2}\left(\delta_{\beta \gamma} h_{\alpha \delta \epsilon \zeta}^{2}(\underline{\boldsymbol{y}})+\delta_{\alpha \gamma} h_{\beta \delta \epsilon \zeta}^{2}(\underline{\boldsymbol{y}})\right),
$$

and $\underline{\nabla}_{Y} \boldsymbol{K}$ is the second gradient of the macroscopic strain $\left(\left(\underline{\nabla}_{Y} \boldsymbol{K}\right)_{\alpha \beta \gamma \delta}=\left(\partial_{Y_{\delta}} K_{\alpha \beta \gamma}\right)\right)$. The corresponding approximation of the strain energy average $\left\langle\frac{1}{2} \varepsilon^{\eta}: c: \varepsilon^{\eta}\right\rangle$ on the unit-cell writes

$$
\frac{1}{2}\left[\boldsymbol{E}: \boldsymbol{C}: \boldsymbol{E}+\eta^{2}\left(\boldsymbol{K}^{\top}: \boldsymbol{F}: \boldsymbol{K}+2 \boldsymbol{E}: \boldsymbol{G}:\left(\underline{\boldsymbol{\nabla}}_{Y} \boldsymbol{K}\right)\right)+\eta^{4}\left(\underline{\boldsymbol{\nabla}}_{Y} \boldsymbol{K}\right)^{\top}: \boldsymbol{H}^{\#}:\left(\underline{\boldsymbol{\nabla}}_{Y} \boldsymbol{K}\right)\right]
$$

$$
{ }^{2} \text { Indeed: }\left\langle\boldsymbol{a}^{1^{\top}}: \boldsymbol{c}: \boldsymbol{a}^{1}\right\rangle=\left\langle\left(\underline{\nabla}_{y}^{s} \boldsymbol{h}^{1}\right)^{\top}: \boldsymbol{c}: \boldsymbol{a}^{1}+\boldsymbol{c}: \boldsymbol{a}^{1}\right\rangle=\left\langle-\boldsymbol{h}^{1^{\top}} \cdot \underline{\operatorname{div}}_{y}\left(\boldsymbol{c}: \boldsymbol{a}^{1}\right)\right\rangle+\left\langle\boldsymbol{c}: \boldsymbol{a}^{1}\right\rangle=\left\langle\boldsymbol{c}: \boldsymbol{a}^{1}\right\rangle
$$


where the sixth-order stiffness tensors $\boldsymbol{F}, \boldsymbol{G}$ and the eight-order stiffness tensor $\boldsymbol{H}^{\#}$ are defined by:

$$
\boldsymbol{F}=\left\langle\boldsymbol{a}^{2^{\top}}: \boldsymbol{c}: \boldsymbol{a}^{2}\right\rangle, \quad \boldsymbol{G}=\left\langle\boldsymbol{a}^{1^{\top}}: \boldsymbol{c}: \boldsymbol{p}^{2}\right\rangle \quad \text { and } \quad \boldsymbol{H}^{\#}=\left\langle\boldsymbol{p}^{2^{\top}}: \boldsymbol{c}: \boldsymbol{p}^{2}\right\rangle
$$

Again, odd-order tensors vanish here thanks to the centrosymmetry assumption for the unit-cell. The form (33) of the homogenized energy involves the third-gradient of the displacement $\boldsymbol{\nabla}_{Y} \boldsymbol{K}$. At order $\eta^{2}$, this dependence can be removed through an integration by parts on the macroscopic domain of the cross term $\boldsymbol{E}: \boldsymbol{G}:\left(\underline{\boldsymbol{\nabla}}_{Y} \boldsymbol{K}\right)$. Ignoring boundary terms and focusing on the bulk energy, we obtain the following average strain energy density:

$$
\frac{1}{2}\left[\boldsymbol{E}: \boldsymbol{C}: \boldsymbol{E}+\eta^{2} \boldsymbol{K}^{\top}:\left(\boldsymbol{F}-\boldsymbol{G}-\boldsymbol{G}^{\boldsymbol{\top}}\right): \boldsymbol{K}+\eta^{4}\left(\underline{\nabla}_{Y} \boldsymbol{K}\right)^{\top}: \boldsymbol{H}:\left(\underline{\boldsymbol{\nabla}}_{Y} \boldsymbol{K}\right)\right] .
$$

Like in the previous truncations, the highest order term in this energy is not reliable as the error committed when approximating $\varepsilon^{\eta}(\underline{\boldsymbol{y}}, \underline{\boldsymbol{Y}})$ is of order $\eta^{2}$. Hence we can only rely on the following approximation of the average energy density:

$$
\frac{1}{2}\left[\boldsymbol{E}: \boldsymbol{C}: \boldsymbol{E}+\eta^{2} \boldsymbol{K}^{\top}:\left(\boldsymbol{F}-\boldsymbol{G}-\boldsymbol{G}^{\boldsymbol{\top}}\right): \boldsymbol{K}\right] .
$$

The sixth-order tensor:

$$
\boldsymbol{D}=\boldsymbol{F}-\boldsymbol{G}-\boldsymbol{G}^{\top}
$$

characterizes strain-gradient effects. Note that $\boldsymbol{D}$ may not be positive in which case the present energy cannot be used as an elastic energy.

The higher-order convergence result from Smyshlyaev and Cherednichenko (2000). This sequence of approximations for the averaged energy may be continued for higher-order truncation of the displacement $\underline{\boldsymbol{u}}^{\eta}$. They are expected to provide improved homogenized approximations when the scale separation is not completely satisfied. Indeed Smyshlyaev and Cherednichenko (2000) proved a convergence result showing that the use of the averaged energy obtained by truncating $\underline{\boldsymbol{u}}^{\eta}$ at order $p$ provides an approximation of the "actual displacement" with an error of order $\eta^{p}$. In this result the "actual displacement" must be understood as an average of the actual equilibrium displacement for all possible positions of the microstructure with respect to the macroscopic domain. Indeed, the exact position of the microstructure is generally not known. Contrary to the classical leading-order homogenized approximation where this effect may be neglected, seeking higher-order estimates requires to remove this influence by averaging.

However the result Smyshlyaev and Cherednichenko (2000) must be evoked with caution in our context. Indeed it has been established under conditions which are not satisfied by the structures we are considering. First, the authors consider only a boundary value problem with periodic boundary conditions. This eliminates any boundary layer effect which can pollute higher-order estimates. Secondly and more importantly, they consider only microstructures with a finite elastic contrast (thus voids and 
rigid inclusions are forbidden). This ensures in particular that $\boldsymbol{C}$ is positive definite and bounded. This theoretical result was investigated numerically on periodic circular inclusions by Ameen et al. (2018) who confirmed the improved estimates when the scale separation is low and even with rather large contrast. However, for the microstructure they considered, scale effects were only observed for $\eta \sim 1 / 3$ which questions even the relevance of deriving a homogenized model and confirms the robustness of the classical homogenization method.

Indeed, preserving contributions of order $\eta^{2}$ in energy (36) requires that $\eta^{2} \boldsymbol{D}$ is somehow comparable to $\boldsymbol{C}$. This implies that either $\boldsymbol{D}$ is large, either $\boldsymbol{C}$ is small, at least in some common directions. That can be achieved only if $\boldsymbol{c}$ is large (even infinite) in some part of $\mathcal{Y}$ and small (or vanishing) in some other part. One way to achieve this is to consider microstructures featuring floppy modes which corresponds to the case where $\boldsymbol{C}$ becomes ill-conditioned. This is the case of the pantographic material described in Section 2.1 and investigated in the following section. This case falls out the classical homogenization theory with finite contrast and out of the framework assumed in (Smyshlyaev and Cherednichenko, 2000).

\section{Homogenization of the pantographic material}

We now consider the pantographic material described in Section 2.1, parameterized by the junction thinness $\rho$. The domain of the microstructure domain is changing with $\rho$ and we numerically investigate the case $\rho \rightarrow 0$.

The strain-gradient homogenization scheme described in the previous section is implemented in the python package HO-homog (Durand and Lebée, 2020) (open source project). HO-homog is specially designed to automate the numerical application of higher-order homogenization schemes such as the one presented here. It is based on the library Dolfin (Logg et al., 2012) for solving the auxiliary problems with the finite element method and for performing operations on the solutions fields $\underline{\boldsymbol{u}}^{k}, \boldsymbol{\varepsilon}^{k}$ and $\boldsymbol{\sigma}^{k}$. It also includes features for parametric modeling of $2 \mathrm{D}$ unit-cell geometries like the one shown in Figure 1 and generating the associated finite element meshes. For these functionalities HO-homog makes use of Gmsh (Geuzaine and Remacle, 2009), a finite-element mesh generator. As junctions play an essential role in the behavior of this metamaterial, we impose a significantly higher mesh density in the vicinity of the junctions: the characteristic length of the elements varies from $a / 4$ in the area far from any junction to $\rho a / 8$ within small areas around each junction (Figure 1).

We solve the auxiliary problems (8) and (18) numerically and we thus obtain finite element functions that represent the localization tensors $\boldsymbol{h}^{1}, \boldsymbol{h}^{2}, \boldsymbol{a}^{1}, \boldsymbol{a}^{2}$. Then, applying the formula (17), (34) and (37) results in the numerical values of the stiffness tensors for the first-gradient equivalent medium $\left(\boldsymbol{C}^{\rho}\right)$ and the strain-gradient equivalent medium $\left(\boldsymbol{C}^{\rho}\right.$ and $\left.\boldsymbol{D}^{\rho}\right)$. Note that shifting the microstructure in Direction 1 or 2 does not change the values for $\boldsymbol{C}^{\rho}$ and $\boldsymbol{D}^{\rho}$ : the localization tensors are the same regardless of the shift of coordinates. Note also that considering a larger unit-cell (made of several 
minimal unit-cells) does not change the homogenized tensors either.

\subsection{First-order auxiliary problem}

Corrector fields. Figure 4 presents the displacement field $\underline{\boldsymbol{u}}^{E}$ defined as:

$$
\underline{\boldsymbol{u}}^{E}=\boldsymbol{E} \cdot \underline{\boldsymbol{y}}+\eta \boldsymbol{h}^{1}(\underline{\boldsymbol{y}}): \boldsymbol{E},
$$

for a junction thinness $\rho=0.05$ and for each unit strain case: $E_{11}, E_{22}$ and $E_{12}$. The norm of the corresponding local strain field, $\left\|\boldsymbol{a}^{1}(\underline{\boldsymbol{y}}): \boldsymbol{E}\right\|$, is plotted on the deformed configuration. Note that $\boldsymbol{h}^{1}$, like all other localization tensors, depends on $\rho$, even though this dependence is not recalled in our notation.

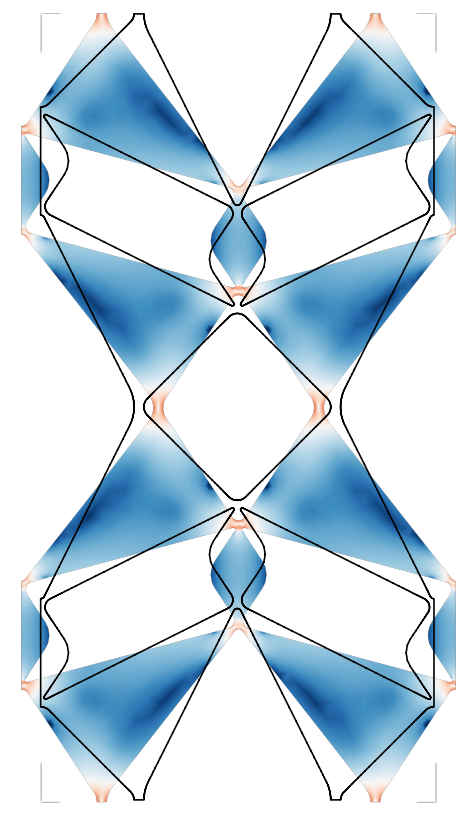

$E_{11}$

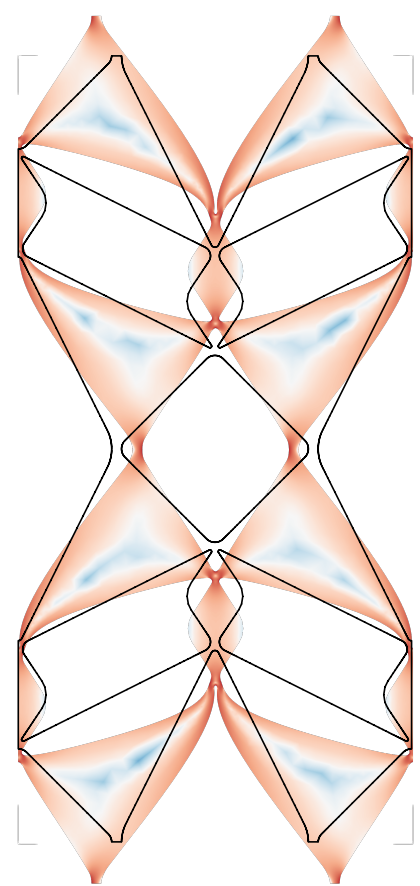

$E_{22}$

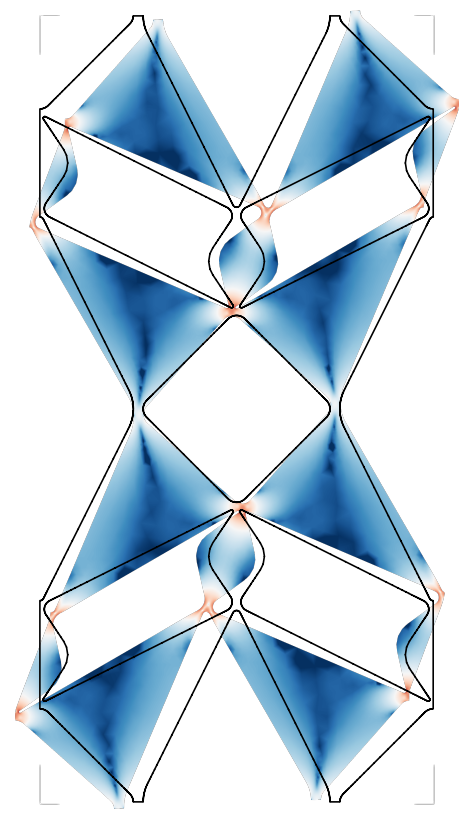

local strain,
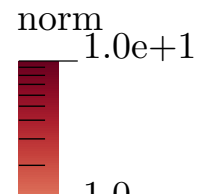

$E_{12}$

Figure 4: Displacement and strain fields produced by each unit macroscopic strain component when $\rho=0.05$. Scale factors of the deformed shapes: 0.1 for $E_{11}$ and $E_{22}, 0.07$ for $E_{12}$.

It appears that both macroscopic strains $E_{11}$ and $E_{12}$ essentially induce relative rotations of the triangles and rhombi inside the unit-cell, while strain is concentrated at junctions. The stretch $E_{11}$ corresponds to the pantographic mode and the shear $E_{12}$ is also almost free thanks to rhombi which allow relative horizontal motion between two pantograph strips (Figure 1). Hence these macroscopic strains appear as floppy modes of the microstructure. On the contrary, the macroscopic strain $E_{22}$ generates significant strains in the triangles and corresponds to a stiff mode.

When $\rho \rightarrow 0$, the scaling of strain and stress in the vicinity of the junctions can be more precisely described. Indeed, the triangles reaching the junction may be treated as wedges loaded at the tip (see Figure 5). For the stiff mode $E_{22}$ triangles are loaded with concentrated forces flowing through junctions 


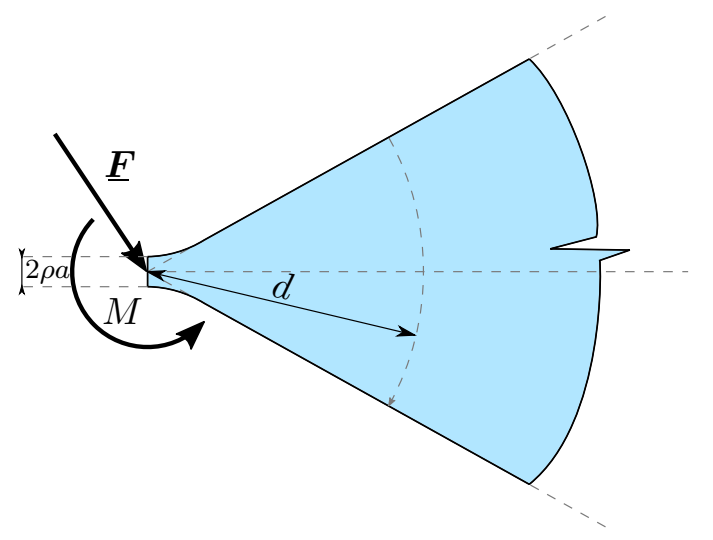

Figure 5: Wedge with concentrated load and couple at the tip.

because of incompatible relative motions of the triangles. This corresponds to the well-known Flamant (1892) solution where the stress distribution inside the wedge scales as $d^{-1}$ where $d$ is the distance to the tip (Figure 5). For the floppy modes $E_{11}$ and $E_{12}$ the corresponding loading of the wedge is a concentrated couple at the tip. Similar arguments based on Michell (1899) general solution indicate that, in this case, the stress distribution in the wedge scales as $d^{-2}$.

First-order homogenized stiffness. Scaling of the stress close to junctions have a direct influence on the total energy in the unit-cell and consequently on the homogenized stiffness. For a floppy mode, as the stress in each branch of the triangles scales as $d^{-2}$ close to all junctions, it is possible to argue that the corresponding overall strain energy in the unit-cell scales as $\rho^{2}$ in the auxiliary problem. Similar arguments also indicate that for a stiff mode, as the stress scales as $d^{-1}$, the overall strain energy scales as $\frac{1}{|\log \rho|}$.

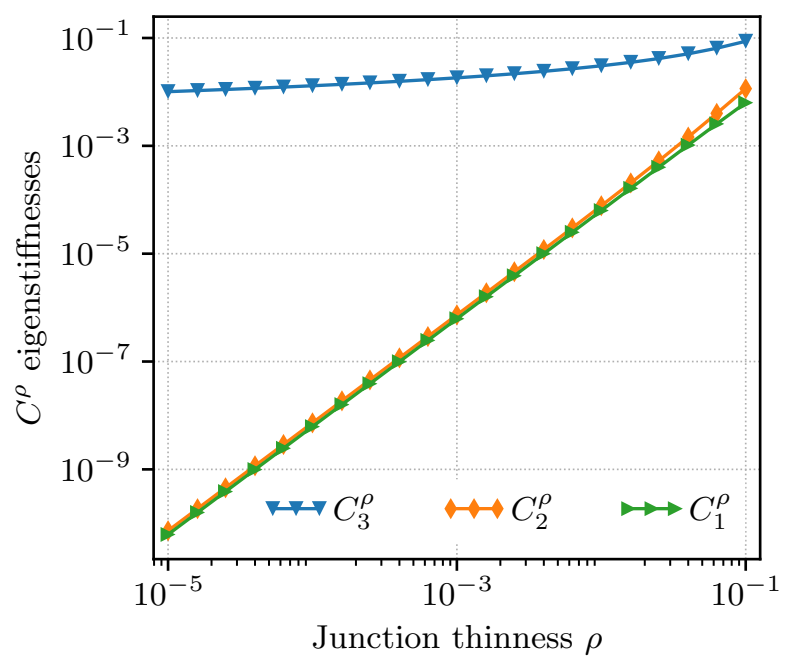

Figure 6: Eigenstiffnesses of the first-order stiffness tensor $\boldsymbol{C}^{\rho}$ as functions of the junction thinness

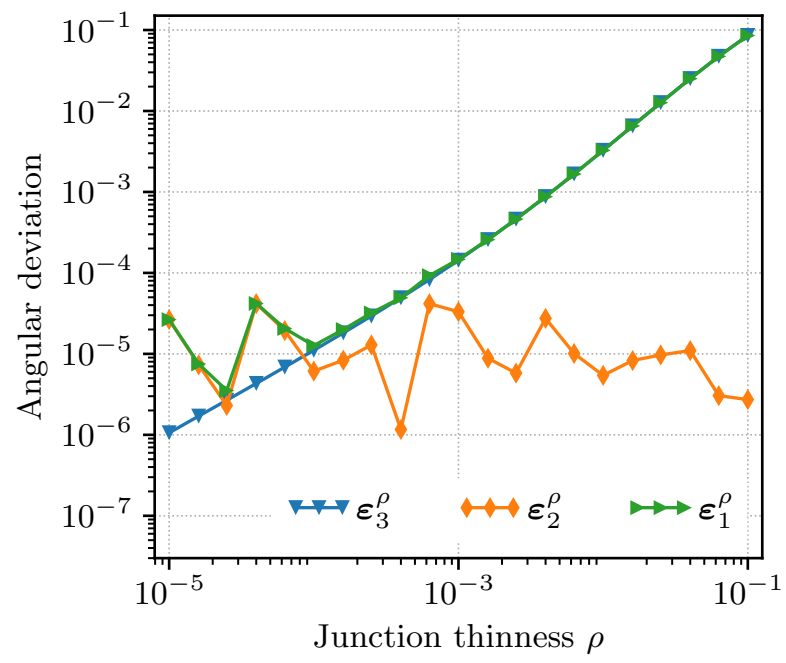

Figure 7: Angular deviation of the eigenstrains of the firstorder stiffness tensor $\boldsymbol{C}^{\rho}$ from their limit eigenstrains when $\rho \rightarrow 0$. 
This is confirmed when considering the diagonal form of $\boldsymbol{C}^{\rho}$ :

$$
C^{\rho}=\sum_{p=1}^{3} C_{p}^{\rho} \varepsilon_{p}^{\rho} \otimes \varepsilon_{p}^{\rho} \quad \text { where } \quad \varepsilon_{p}^{\rho}: \varepsilon_{q}^{\rho}=\delta_{p q},
$$

introducing the eigenvalues of $\boldsymbol{C}^{\rho},\left(C_{1}^{\rho}, C_{2}^{\rho}, C_{3}^{\rho}\right)$ and the orthonormal basis $\left(\varepsilon_{1}^{\rho}, \varepsilon_{2}^{\rho}, \varepsilon_{3}^{\rho}\right)$ of the associated eigenvectors. In this context of elasticity, we will call $C_{p}^{\rho}$ eigenstiffnesses and $\varepsilon_{p}^{\rho}$ eigenstrains.

The three eigenstiffnesses $C_{p}^{\rho}$ are sorted in ascending order and plotted in Figure 6 as functions of the junction thinness. It appears that two eigenstiffnesses converge to 0 at a rate of $\rho^{2}$ : they correspond to floppy modes. The last eigenstiffness $C_{3}^{\rho}$ convergence is much lower and similar to

$\frac{1}{|\log \rho|}$. This is the stiff mode. The corresponding eigenstrains converge respectively toward $\varepsilon_{1}^{0}=E_{11}$, $\varepsilon_{2}^{0}=\sqrt{2} E_{12}$ and $\varepsilon_{3}^{0}=E_{22}$. Indeed Figure 7 presents the angular deviation $\sin \left(\theta_{p}^{\rho}\right)=\sqrt{1-\left(\varepsilon_{p}^{\rho}: \varepsilon_{p}^{0}\right)^{2}}$ with respect to these limits.

Note that, for $\rho=0.02$, the floppy modes eigenstiffnesses are already two orders of magnitude smaller than the stiff mode, showing the efficiency of compliant junctions. Consequently, when $\rho$ is reasonably small, we can define without ambiguity the vector space of floppy modes of $\boldsymbol{C}^{\rho}$ as $\operatorname{span}\left\{\varepsilon_{1}^{\rho}, \varepsilon_{2}^{\rho}\right\}$. When the strain belongs to this space, the associated first-order energy is so small that the contributions from the second-order $\eta^{2} \boldsymbol{D}$ may become comparable. These contributions are investigated in the next subsection.

\subsection{Second-order auxiliary problem}

Corrector fields. Figure 8 presents the total displacement around a unit-cell computed for a uniform macroscopic strain-gradient $\boldsymbol{K}$. Indeed, considering a quadratic variation of the macroscopic displacement $\boldsymbol{U}$ in the expansion (23) yields the following approximation of the displacement:

$$
\underline{\boldsymbol{u}}^{K}=\frac{1}{2} \widetilde{\boldsymbol{K}}:(\underline{\boldsymbol{y}} \otimes \underline{\boldsymbol{y}})+\eta \boldsymbol{h}^{1}(\underline{\boldsymbol{y}}):(\boldsymbol{K} \cdot \underline{\boldsymbol{y}})+\eta^{2} \boldsymbol{h}^{2}(\underline{\boldsymbol{y}}): \boldsymbol{K},
$$

where $\widetilde{\boldsymbol{K}}$ is the second-gradient of the displacement corresponding to the strain-gradient $\boldsymbol{K}$. It is easily written in terms of $\boldsymbol{K}$ as follows ${ }^{3}$ :

$$
\begin{aligned}
& \widetilde{K}_{111}=K_{111}, \quad \widetilde{K}_{221}=\widetilde{K}_{212}=K_{221}, \quad \widetilde{K}_{122}=2 K_{122}-K_{221}, \\
& \widetilde{K}_{222}=K_{222}, \quad \widetilde{K}_{112}=\widetilde{K}_{121}=K_{112}, \quad \widetilde{K}_{211}=2 K_{211}-K_{112} .
\end{aligned}
$$

Second-order corrector fields may be interpreted as the way the microstructure responses to linear variations of the macroscopic strains. This is well illustrated with the present pantographic material. For instance, $K_{112}$ corresponds clearly to a beam-like bending of the pantograph strips. Similarly, $K_{111}$ reveals the incompatibility of the rotation of triangles when the floppy stretch $E_{11}$ is not uniform.

The correctors associated to all strain-gradient components, except $K_{122}$, feature significant deformation of the solid triangles corresponding to stiff strain-gradient modes. The corrector associated

$$
{ }^{3} \text { i.e.: } \underline{\nabla}_{y} \boldsymbol{\nabla}_{y}^{s}\left(\frac{1}{2} \tilde{\boldsymbol{K}}: \underline{\boldsymbol{y}} \otimes \underline{\boldsymbol{y}}\right)=\boldsymbol{K}
$$




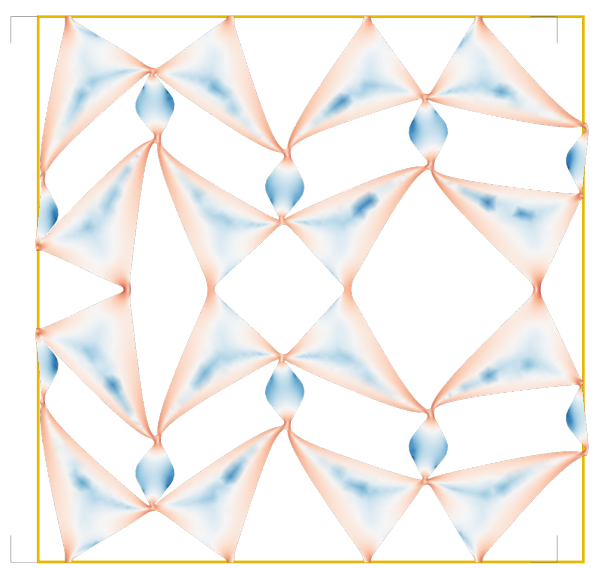

$K_{111}$

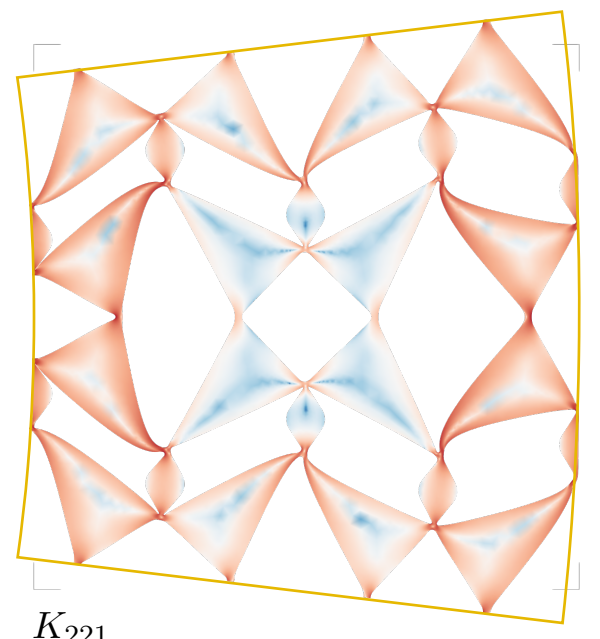

$K_{221}$

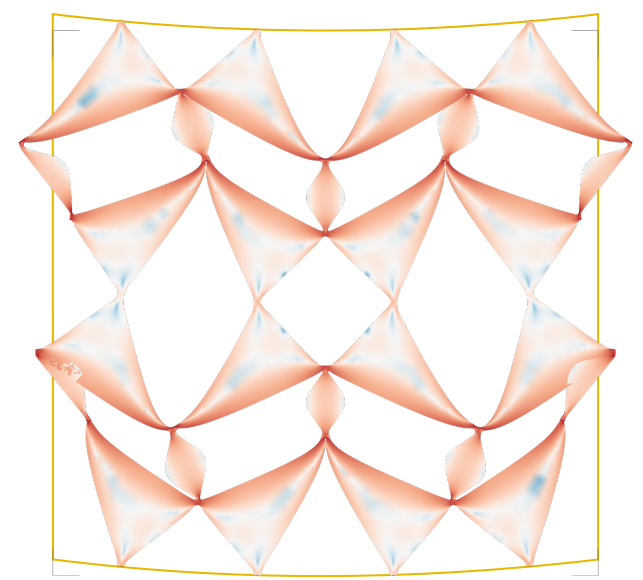

$K_{121}$

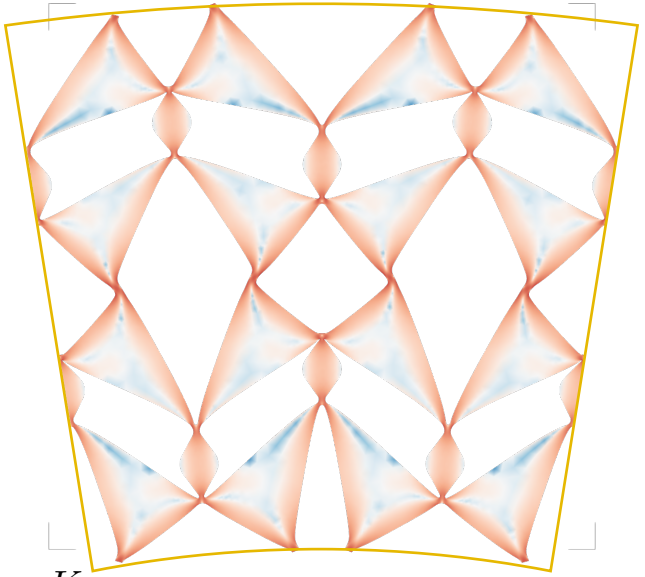

$K_{112}$

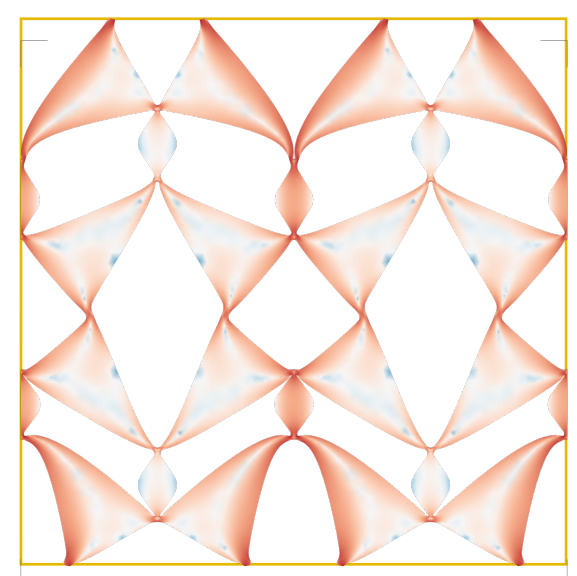

$K_{222}$

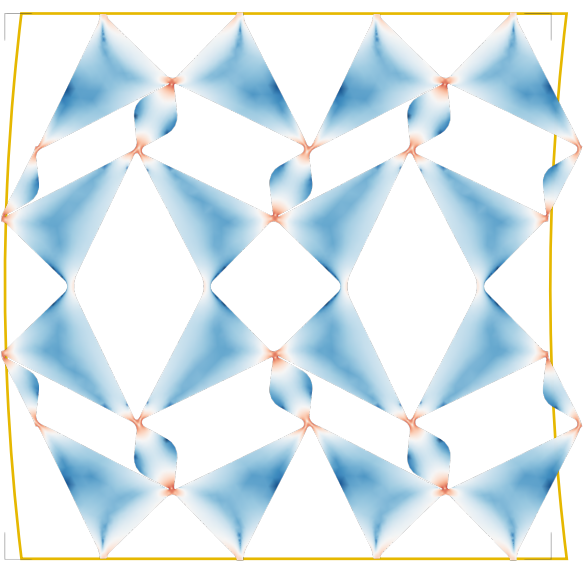

$K_{122}$

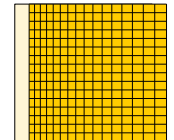

$K_{111}$

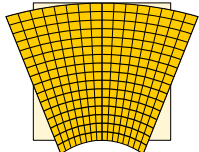

$K_{112}$

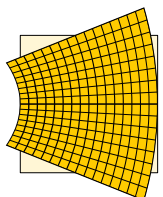

$K_{221}$

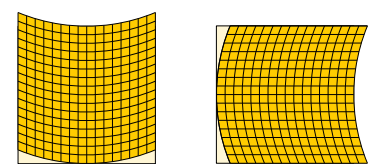

$K_{121} \quad K_{122}$
$K_{222}$

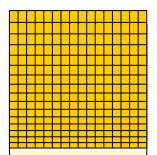

local strain,

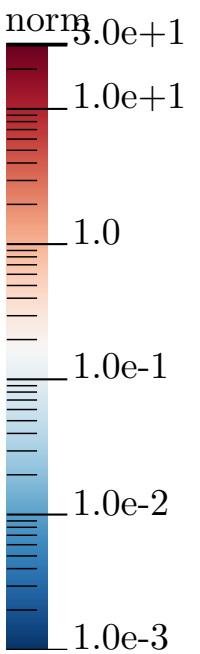

Figure 8: Total displacement and strain field produced by each unit strain-gradient component. On the upper-right corner, the macroscopic deformation corresponding to each strain-gradient component is represented by applying the deformation $\frac{1}{2} \widetilde{\boldsymbol{K}}:(\underline{\boldsymbol{y}} \otimes \underline{\boldsymbol{y}})$ to a regular grid.

to $K_{122}$ corresponds to a mode which remains floppy at the second order. Indeed, recall that the presence of rhombi allows almost zero energy relative motion in Direction 1 between pantograph strips (Figure 1). Hence, all macroscopic displacements of the form $\underline{\boldsymbol{U}}=f\left(x_{2}\right) \underline{\boldsymbol{e}}_{1}$ have almost zero energy. 
Strain-gradient characteristic lengths. We compute now the second-order homogenized stiffness $\boldsymbol{D}^{\rho}$ defined from the two-scale asymptotic expansion in Equation (37). In order to illustrate it, we present the six quantities defined, for $p \in\{1,2,3\}$ and $\alpha \in\{1,2\}$, by

$$
\ell_{p, \alpha}^{\rho}=\eta \sqrt{\frac{\left(\varepsilon_{p \otimes \underline{e}_{\alpha}}^{\rho}\right)^{\top}: \boldsymbol{D}^{\rho}:\left(\varepsilon_{p}^{\rho} \otimes \underline{\boldsymbol{e}}_{\alpha}\right)}{C_{p}^{\rho}}} .
$$

These quantities are six intrinsic lengths of the effective strain-gradient continuum (relative to the size $T$ of the macroscopic domain). Each one corresponds to the response of the material to a variation, in the direction $\underline{e}_{\alpha}$, of a strain remaining proportional to the eigenstrain $\varepsilon_{p}^{\rho}$ : any such variation occurring over a distance of order $\ell_{p, \alpha}^{\rho}$ (or smaller) is associated to a non-negligible elastic energy. The strain-gradient effect is negligible at the macroscopic scale when the intrinsic length is of the order of the relative unit-cell typical size $\eta$. It becomes important when the intrinsic length is of order $1=\eta^{0}$.

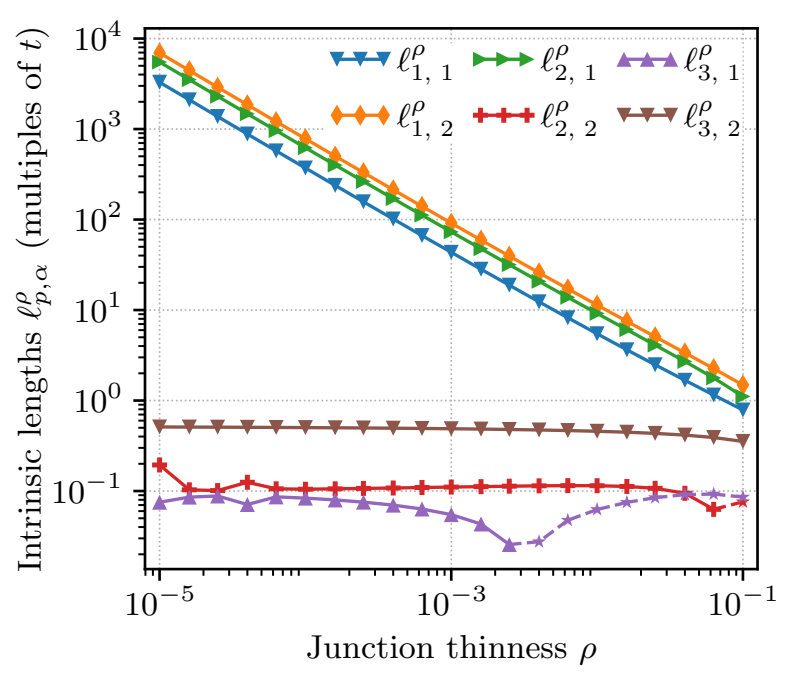

Figure 9: Intrinsic lengths of the equivalent strain-gradient continuum. Note that, as $\boldsymbol{D}^{\rho}$ may be negative, the lengths may be complex : this is the case for $\ell_{3,2}^{\rho}$ when $\rho$ is rather large; then its modulus is plotted using star markers and dotted lines.

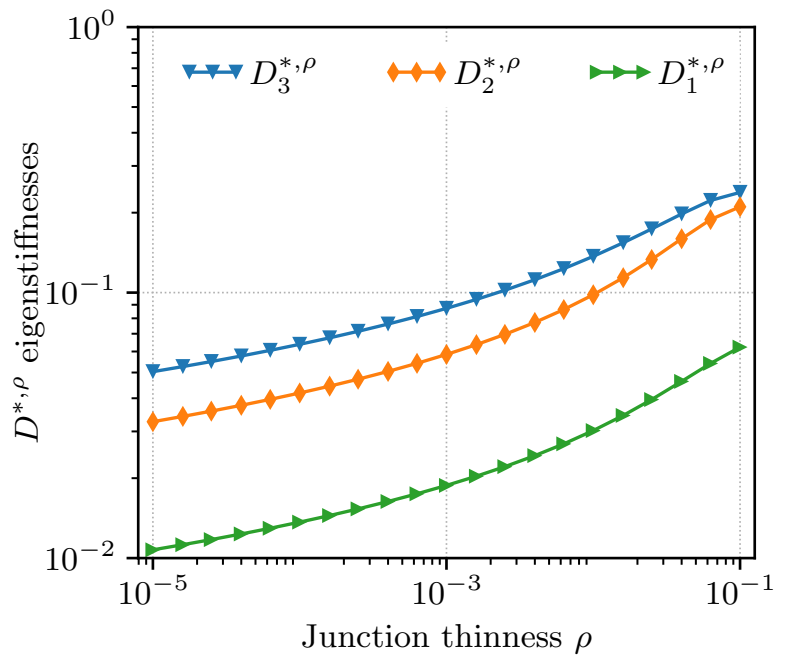

Figure 10: Eigenstiffnesses of $\boldsymbol{D}^{*, \rho}$ obtained after projection.

Figure 9 presents theses characteristic lengths expressed as multiples of $\eta$. It appears clearly that, when junctions are becoming thin, three lengths remain of the order of the cell size or smaller whereas the three others are real and become significantly large compared to it.

The reason why $\ell_{3,1}$ and $\ell_{3,2}$ remain of the order of the cell size is the following : they are related to the stiff strain-gradient modes $K_{221}$ and $K_{222}$ (Figure 8 ) associated to the stiff strain mode $\varepsilon_{3}^{\rho}$. The energy of these modes is expected to scale as $\frac{1}{|\log \rho|}$ exactly like the first-order eigenstiffness $C_{3}^{\rho}$. The reason why $\ell_{2,2}$ also remains of the order of the cell size is similar but with a different energy scaling : it is related to the floppy strain-gradient mode $K_{122}$ (Figure 8) associated to the floppy strain mode $C_{2}^{\rho}$. The energies corresponding to theses two modes share again the same scaling which is now $\rho^{2}$. The 
situation is different for the other lengths $\ell_{1,1}, \ell_{1,2}$ and $\ell_{2,1}$ : they correspond to stiff strain-gradient modes whose energy scales as $\frac{1}{|\log \rho|}$ associated to floppy strain modes whose energy scales as $\rho^{2}$. It is then not surprising that these lengths scale as $\frac{1}{\rho \sqrt{|\log \rho|}}$.

For $\rho$ small enough, $\frac{1}{\rho \sqrt{|\log \rho|}}$ may become comparable to $1 / \eta$, revealing strain-gradient effects which are significant from the macroscopic point of view.

It is also important to understand why the lengths associated with floppy strain modes are real. Indeed, in Equation (37), unsigned contributions come from $\boldsymbol{G}^{\rho}$ and correspond to cross energies between the first-gradient strain localization $\boldsymbol{a}^{1}$ and the second-gradient displacement corrector $\boldsymbol{h}^{2}$. When a floppy strain mode is considered, the corresponding strain localization $\boldsymbol{a}^{1}$ becomes small when $\rho \rightarrow 0$ and contributions from $\boldsymbol{G}^{\rho}$ become negligible compared to those from $\boldsymbol{F}^{\rho}$ in Equation (37).

\subsection{Strain-gradient model for the pantographic material}

From the behavior of the characteristic lengths as function of $\rho$, we conclude that, $\boldsymbol{D}^{\rho}$ cannot be used directly for describing the strain-gradient effective behavior of the pantographic material. Indeed, contributions with a characteristic length which remains of the order of the cell size should be discarded in a macroscopic model. Furthermore, $\boldsymbol{D}^{\rho}$ may not be positive definite. In that case, deriving a well-posed model would require some regularization by adding for instance third-gradient terms in the homogenized energy, terms whose physical bases would have to be justified. That is why we suggest to keep in the macroscopic effective model only the strain-gradient terms which correspond to macroscopic intrinsic lengths.

The second-order stiffness tensor for the pantographic material. We suggest projecting $\boldsymbol{D}^{\rho}$ on the subspace of the strain gradients which present significant characteristic lengths, namely on the space spanned by $\varepsilon_{1}^{\rho} \otimes \underline{\boldsymbol{e}}_{1}, \varepsilon_{1}^{\rho} \otimes \underline{\boldsymbol{e}}_{2}, \varepsilon_{2}^{\rho} \otimes \underline{\boldsymbol{e}}_{1}$ corresponding to the lengths $\ell_{1,1}^{\rho}, \ell_{1,2}^{\rho}$ and $\ell_{2,1}^{\rho}$. We set

$$
\boldsymbol{D}^{* \rho}=\boldsymbol{P}^{\rho}: \boldsymbol{D}^{\rho}: \boldsymbol{P}^{\rho}
$$

where $\boldsymbol{P}^{\rho}=\varepsilon_{1}^{\rho} \otimes \underline{\boldsymbol{e}}_{1} \otimes \underline{\boldsymbol{e}}_{1} \otimes \varepsilon_{1}^{\rho}+\varepsilon_{1}^{\rho} \otimes \underline{\boldsymbol{e}}_{2} \otimes \underline{\boldsymbol{e}}_{2} \otimes \varepsilon_{1}^{\rho}+\boldsymbol{\varepsilon}_{2}^{\rho} \otimes \underline{\boldsymbol{e}}_{1} \otimes \underline{\boldsymbol{e}}_{1} \otimes \varepsilon_{2}^{\rho}$. As already pointed out, this projection ensures that $\boldsymbol{D}^{* \rho}$ is positive for $\rho$ sufficiently small, in addition to removing spurious contributions from short characteristic lengths. This is illustrated in Figure 10 which shows the three eigenstiffnesses of $\boldsymbol{D}^{* \rho}$.

Homogenized energy. From the previous developments we arrive at the following homogenized straingradient energy density:

$$
W^{\rho, \eta}(\underline{\boldsymbol{U}})=\frac{1}{2}\left(\boldsymbol{E}: \boldsymbol{C}^{\rho}: \boldsymbol{E}+\eta^{2} \boldsymbol{K}^{\top}: \boldsymbol{D}^{* \rho}: \boldsymbol{K}\right)
$$

Next section is devoted to checking that this strain-gradient model actually describes in a very accurate way the macroscopic behavior of the considered pantographic material. 


\section{Comparison with full-scale simulations and empirical error estimates}

Several numerical campaigns have been performed in order to check the accuracy of the proposed strain-gradient model for the pantographic metamaterial.

The results of the first campaign have already been presented in Figure 3 where one can qualitatively observe a huge difference between the accuracy provided by the proposed effective strain-gradient model and the accuracy of the classical first-gradient model effective model. On this graph, solid lines represent the closed-form displacement solution $\underline{U}^{\mathrm{SG}}$ that corresponds to the problem where the pantographic metamaterial is replaced by the proposed strain-gradient equivalent homogeneous medium, with the elasticity characteristics calculated using the method presented in Sections 3 and 4 . This macroscopic approximate solution $\underline{\boldsymbol{U}}^{\mathrm{SG}}$ fits perfectly the results of the full-scale simulations, when the junctions are thin $\left(\rho \leq 1 \times 10^{-3}\right)$ as well as when they are thick $\left(\rho=1 \times 10^{-1}\right)$, while significant discrepancies can be noted when using the first-gradient homogeneous equivalent medium. We do not detail here the computation of the equilibrium solution $\underline{U}^{\mathrm{SG}}$ nor the way we perform the full-scale simulation. Indeed we prefer to focus on a slightly different benchmark problem which is more suitable for a quantitative comparison of the accuracy of the two models. The computation of the equilibrium solution $\underline{U}^{\text {SG }}$ and the way we perform the full-scale simulation are totally similar in both problems. We did not choose to present this new benchmark problem in Section 2.2 as it generates a lower discrepancy between first-gradient and strain-gradient approximations and thus does not provide a so glaring evidence of the superiority of the strain-gradient effective model. We focus on it now because it is better conditioned for the full-scale solution.

\subsection{Benchmark problem}

The considered material is still a plate made of the pantographic metamaterial and infinite in Direction 2. It is now clamped on the left-hand side $x_{1}=0$ and subjected to a prescribed displacement boundary condition $\underline{\boldsymbol{u}}=u^{d} \underline{\boldsymbol{e}}_{1}$ on its right-hand boundary at $x_{1}=T$. There is no body force.

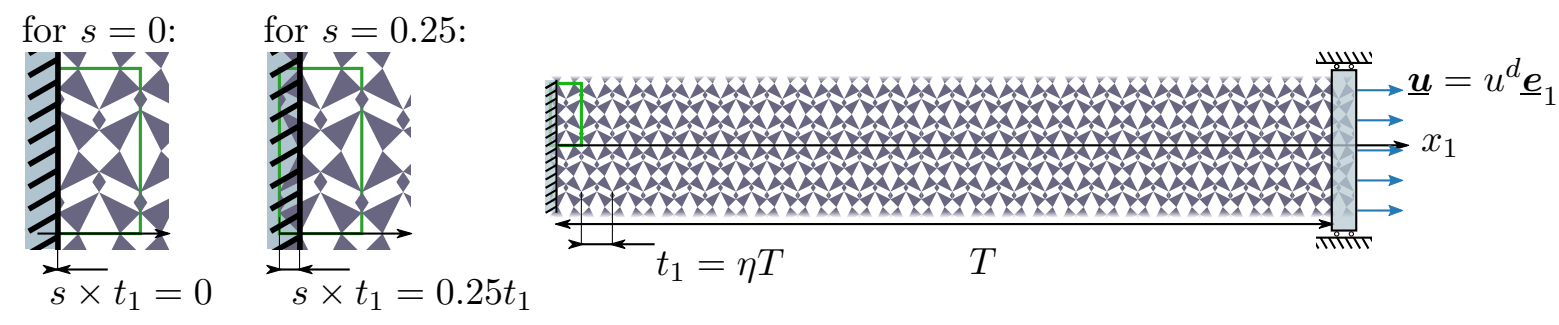

Figure 11: Second benchmark elasticity problem, used for the comparison between the first-gradient model, the proposed strain-gradient model and full-scale simulations.

Furthermore, we consider several configurations that correspond to shifts of the microstructure in Direction 1 with respect to the domain. For each configuration, the position of the microstructure is 
measured with $s$, defined on Figure 11. The case $s=0.00$ is the situation ${ }^{4}$ where the boundaries of the domain coincide with the edges of the unit-cell as defined in Section 1 and shown in green in Figure 11.

Thus, the effect of three parameters are investigated: the junction thinness $\rho$, the scale ratio $\eta$ and the microstructure relative position $s$. The first study focuses on the effects of microstructure position $s$, while the second focuses on the separation of scales. The influence of the junction thinness $\rho$ is investigated in both studies.

\subsection{Reference solution by full-scale numerical simulation}

A full-scale finite element simulation is performed with the geometry of the whole microstructure explicitly meshed and using a standard displacement-based finite element formulation. This solution serves as the reference for the following comparisons. $\underline{\boldsymbol{u}}^{\mathrm{fs}}$ and $\varepsilon^{\mathrm{fs}}$ are respectively its equilibrium displacement field and the associated strain field. Quadratic Lagrange triangles are used for the discretization of the displacement field. We use the Python package Dolfin (Logg et al., 2012), a component of the FEniCS Project (Alnæs et al., 2015), for all finite-element calculations presented in this paper. The discrete problem is solved using a direct solver (mumps). Owing to the periodicity of the benchmark problem in Direction 2, it is enough to perform the numerical simulations on a strip $\Omega^{\mathrm{fs}}$ of unit-cells that contains a single unit-cell in Direction 2 and to impose periodic boundary conditions between top and bottom boundaries of $\Omega^{\mathrm{fs}}$. The CAD modeling of $\Omega^{\mathrm{fs}}$ and the generation of the associated mesh are done using Gmsh (Geuzaine and Remacle, 2009). The mesh of the strip is generated by duplicating the mesh of one unit-cell, the same mesh as that used for the homogenization step in Section 4. This meshing strategy has been validated by a convergence study (for $\eta=1 / 20$ ).

\subsection{Approximate solutions from homogenization}

The first-gradient model and the strain-gradient homogenized energies have been computed in Section 4. Computing the equilibrium solution corresponding to these two models is straightforward. In a further step, we can reconstruct the microscopic-scale fluctuations by using the localization tensor fields.

Macroscopic-scale boundary value problems. The first-gradient boundary value problem reduces to a one-dimensional problem $\underline{\boldsymbol{U}}(\underline{\boldsymbol{Y}})=\left(U_{1}\left(Y_{1}\right), U_{2}\left(Y_{1}\right)\right)$ with $\underline{\boldsymbol{U}}=\underline{\mathbf{0}}$ on the left-hand side $Y_{1}=0$ and $\underline{\boldsymbol{U}}=\frac{u^{d}}{T} \underline{\boldsymbol{e}}_{1}$ on the right-hand side $Y_{1}=1$. The equilibrium displacement field $\underline{\boldsymbol{U}}^{\mathrm{C}}$ is simply given, for any $Y_{1} \in[0 ; 1]$, by

$$
U_{1}^{\mathrm{C}}(\underline{\boldsymbol{Y}})=\frac{u^{d}}{T} Y_{1} \quad \text { and } \quad U_{2}^{\mathrm{C}}(\underline{\boldsymbol{Y}})=0 .
$$

Only one component of the associated strain field $\boldsymbol{E}^{\mathrm{C}}$ does not vanish, namely $E_{11}^{\mathrm{C}}(\underline{\boldsymbol{Y}})=\frac{u^{d}}{T}$.

\footnotetext{
${ }^{4}$ The particular configuration $s=0.00$ was also used for the simulations presented in Section 2.2.
} 
The second-gradient boundary value problem also reduces to a one-dimensional problem with the boundary conditions $\underline{\boldsymbol{U}}=\underline{\mathbf{0}}, \boldsymbol{E} \cdot \underline{\boldsymbol{e}}_{1}=0$ for $Y_{1}=0$ and $\underline{\boldsymbol{U}}=\frac{u^{d}}{T} \underline{\boldsymbol{e}}_{1}, \boldsymbol{E} \cdot \underline{\boldsymbol{e}}_{1}=0$ for $Y_{1}=1$. A closed-form solution $\underline{\boldsymbol{U}}^{\mathrm{SG}}$ can also be obtained. We get, for any $Y_{1} \in[0 ; 1]$,

$$
U_{1}^{\mathrm{SG}}(\underline{\boldsymbol{Y}})=B\left(\sinh \left(k\left(Y_{1}-\frac{1}{2}\right)\right)-k \cosh \left(\frac{k}{2}\right)\left(Y_{1}-\frac{1}{2}\right)\right)+\frac{u^{d}}{2 T} \quad \text { and } \quad U_{2}^{\mathrm{SG}}(\underline{\boldsymbol{Y}})=0
$$

with $k=\sqrt{\frac{C_{1111}}{D_{11111}}}$ and $B=\frac{u^{d}}{2 k T}\left(\frac{1}{k} \sinh (k / 2)-\frac{1}{2} \cosh (k / 2)\right)^{-1}$. The corresponding macroscopic strain $\boldsymbol{E}^{\mathrm{SG}}$ and strain-gradient $\boldsymbol{K}^{\mathrm{SG}}$ have only one non-zero component, namely $E_{11}^{\mathrm{SG}}=\partial_{1} U_{1}^{\mathrm{SG}}$ and $K_{111}^{\mathrm{SG}}=\partial_{11} U_{1}^{\mathrm{SG}}$.

Assembling of the approximate solutions. The first-gradient model has been obtained by approximating, for any given macroscopic field $\underline{\boldsymbol{U}}$, the displacement field at the microscopic level by the truncated series (26). For this model, the approximations $\underline{u}^{\mathrm{C}}$ and $\varepsilon^{\mathrm{C}}$ of the displacement field and strain field are thus given by:

$$
\begin{aligned}
& \underline{\boldsymbol{u}}^{\mathrm{C}}(\underline{\boldsymbol{x}})=T\left(\underline{\boldsymbol{U}}^{\mathrm{C}}(\underline{\boldsymbol{Y}})+\eta \boldsymbol{h}^{1}(\underline{\boldsymbol{y}}): \boldsymbol{E}^{\mathrm{C}}(\underline{\boldsymbol{Y}})\right) \\
& \boldsymbol{\varepsilon}^{\mathrm{C}}(\underline{\boldsymbol{x}})=\boldsymbol{a}^{1}(\underline{\boldsymbol{y}}): \boldsymbol{E}^{\mathrm{C}}(\underline{\boldsymbol{Y}}) .
\end{aligned}
$$

In a similar manner, the approximate displacement field $\underline{\boldsymbol{u}}^{\mathrm{SG}}$ and strain field $\boldsymbol{\varepsilon}^{\mathrm{SG}}$ associated to the strain-gradient model are:

$$
\begin{aligned}
& \underline{\boldsymbol{u}}^{\mathrm{SG}}(\underline{\boldsymbol{x}})=T\left(\underline{\boldsymbol{U}}^{\mathrm{SG}}(\underline{\boldsymbol{Y}})+\eta \boldsymbol{h}^{1}(\underline{\boldsymbol{y}}): \boldsymbol{E}^{\mathrm{SG}}(\underline{\boldsymbol{Y}})+\eta^{2} \boldsymbol{h}^{2}(\underline{\boldsymbol{y}}): \boldsymbol{K}^{\mathrm{SG}}(\underline{\boldsymbol{Y}})\right) \\
& \boldsymbol{\varepsilon}^{\mathrm{SG}}(\underline{\boldsymbol{x}})=\boldsymbol{a}^{1}(\underline{\boldsymbol{y}}): \boldsymbol{E}^{\mathrm{SG}}(\underline{\boldsymbol{Y}})+\eta \boldsymbol{a}^{2}(\underline{\boldsymbol{y}}): \boldsymbol{K}^{\mathrm{SG}}(\underline{\boldsymbol{Y}}) .
\end{aligned}
$$

\subsection{Error measures}

Finally, the quality of each approximation $\left(\underline{\boldsymbol{u}}^{\mathrm{C}}, \varepsilon^{\mathrm{C}}\right)$ or $\left(\underline{\boldsymbol{u}}^{\mathrm{SG}}, \varepsilon^{\mathrm{SG}}\right)$ is assessed by computing the relative $L^{2}$ distance from the reference solution for both displacement fields. We define ${ }^{5}$

$$
\delta\left(\underline{\boldsymbol{u}}^{\mathrm{C}}\right)=\frac{\left\|\underline{\boldsymbol{u}}^{\mathrm{fs}}-\underline{\boldsymbol{u}}^{\mathrm{C}}\right\|_{2}}{\left\|\underline{\boldsymbol{u}}^{\mathrm{fs}}\right\|_{2}}, \quad \delta\left(\varepsilon^{\mathrm{C}}\right)=\frac{\left\|\varepsilon^{\mathrm{fs}}-\boldsymbol{\varepsilon}^{\mathrm{C}}\right\|_{2}}{\left\|\varepsilon^{\mathrm{fs}}\right\|_{2}}, \quad \delta\left(\underline{\boldsymbol{u}}^{\mathrm{SG}}\right)=\frac{\left\|\underline{\boldsymbol{u}}^{\mathrm{fs}}-\underline{\boldsymbol{u}}^{\mathrm{SG}}\right\|_{2}}{\left\|\underline{\boldsymbol{u}}^{\mathrm{fs}}\right\|_{2}}, \quad \delta\left(\varepsilon^{\mathrm{SG}}\right)=\frac{\left\|\varepsilon^{\mathrm{fs}}-\varepsilon^{\mathrm{SG}}\right\|_{2}}{\left\|\varepsilon^{\mathrm{fs}}\right\|_{2}} .
$$

Note that homogenized models are often compared to adequate averages of the full-scale solution. Here, we measure the "true" error between the reconstructed approximation and the reference solution.

\subsection{Influence of the relative position of the microstructure}

This first comparison campaign focuses on the influence of the microstructure relative position. Owing to the symmetries of the benchmark problem and the unit-cell, the study range for the $s$

\footnotetext{
${ }^{5}$ The $L^{2}$-norm is defined by:

$$
\|\underline{\boldsymbol{u}}\|_{2}=\left(\int_{\Omega} \underline{\boldsymbol{u}} \cdot \underline{\boldsymbol{u}} \mathrm{d} \Omega\right)^{\frac{1}{2}} \text { and }\|\varepsilon\|_{2}=\left(\int_{\Omega} \varepsilon^{T}: \varepsilon \mathrm{d} \Omega\right)^{\frac{1}{2}} .
$$
}




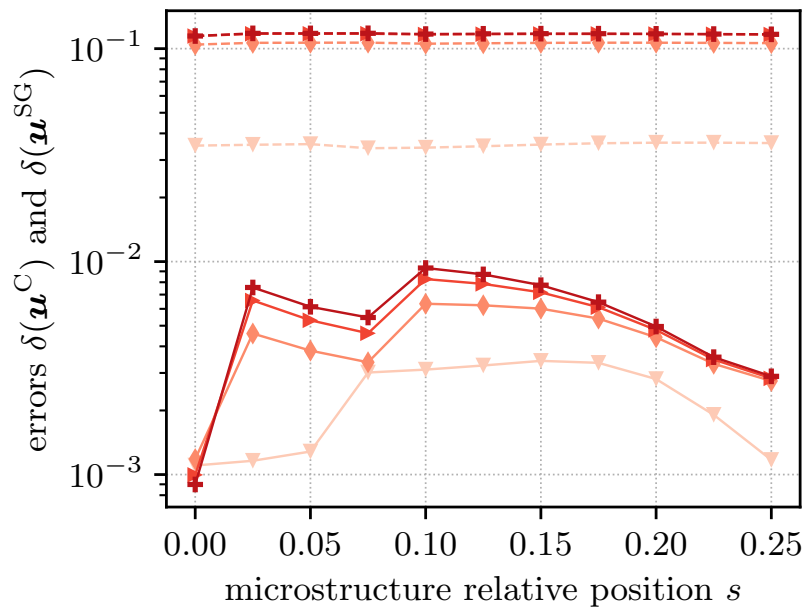

(a)

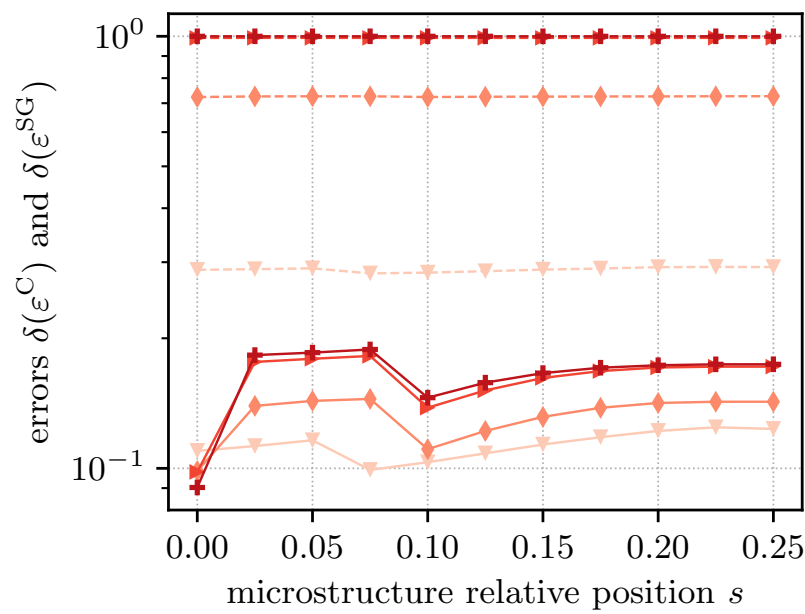

(b)

Junction thinness $\rho$

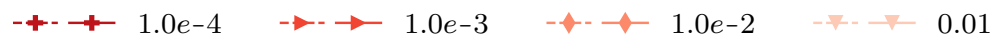

Figure 12: Effects of the relative position of the microstructure $s$ on the accuracy of the first-gradient model (dashed lines) and of the proposed strain-gradient model (solid lines), for $\eta=1 / 20$. The accuracy is measured for both the approximate displacement fields (a) and the approximate strain fields (b).

values can be narrowed to [0.0,0.25]. Eleven values of $s$ are considered, uniformly distributed in this range. We also considered several junction sizes $\rho$ ranging from $10^{-4}$ to $10^{-1}$ and, for this campaign, the scale separation is fixed to $\eta=1 / 20$. This value of $\eta$ is a compromise as it corresponds to a reasonable separation of scale while keeping the calculation cost reasonably low. The comparison process previously described is applied for each of the corresponding triplets $(\rho, \eta, s)$.

Figures $12 \mathrm{a}$ and $12 \mathrm{~b}$ respectively present the errors measured for the approximate displacement fields $\delta\left(\underline{\boldsymbol{u}}^{\mathrm{X}}\right)$ and for the approximate strain fields $\delta\left(\varepsilon^{\mathrm{X}}\right)$ as functions of the microstructure position s. Both approximate solutions, resulting from the proposed strain-gradient model and from the first-gradient model are represented. Solid lines represent the accuracy measure for $\underline{\boldsymbol{u}}^{\mathrm{SG}}$ and $\boldsymbol{\varepsilon}^{\mathrm{SG}}$ while dotted lines correspond to $\underline{\boldsymbol{u}}^{\mathrm{C}}$ and $\boldsymbol{\varepsilon}^{\mathrm{C}}$.

Figure 12a shows that the microstructure relative position $s$ has almost no effect on the accuracy of the approximate solution obtained using the first-gradient model: the overall error $\delta\left(\underline{\boldsymbol{u}}^{\mathrm{C}}\right)$ is so large (about $10^{-1}$ ) that the small fluctuation caused by $s$ is not discernible. The strain-gradient model provides a much more accurate approximate solution and a variation of the microstructure position results in a non-negligible fluctuation of the error $\delta\left(\underline{\boldsymbol{u}}^{\mathrm{SG}}\right)$. It appears that, for a given value of $\rho$, the position $s=0.00$ is the most favorable case and results in the smallest estimation error $\delta\left(\underline{\boldsymbol{u}}^{\mathrm{SG}}\right)$ while $s=0.10$ corresponds to the most unfavorable case. Nonetheless, even for $s=0.10$ the error in terms of displacement with the strain-gradient model is one decade below the error $\delta\left(\underline{u}^{\mathrm{C}}\right)$ which results from the first-gradient model. Moreover, on the considered range of junction thinness $\rho$, the amplitude of the $\delta\left(\underline{\boldsymbol{u}}^{\mathrm{SG}}\right)$ fluctuations due to $s$ become larger when $\rho$ decrease but remains limited, in comparison 


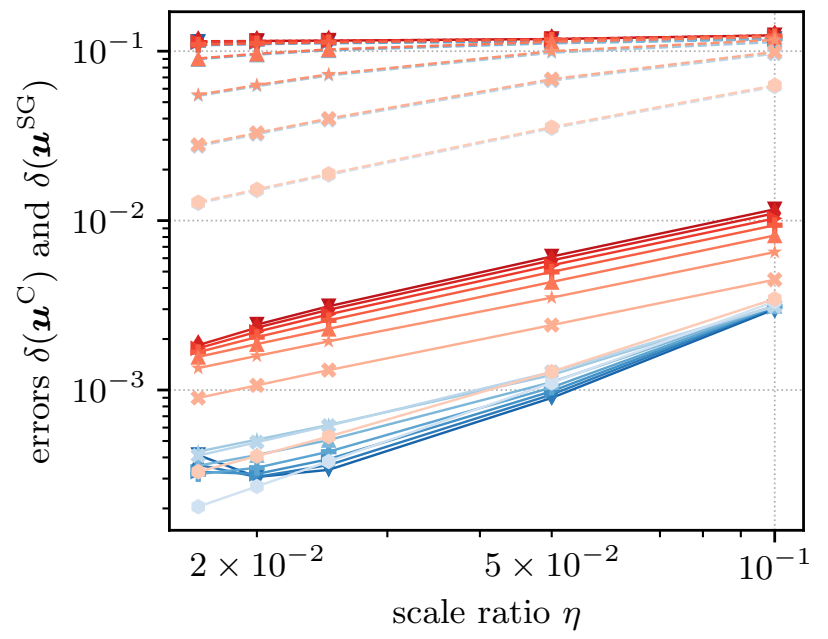

(a)

$$
\begin{aligned}
& \text { for } s=0.00 \text { : } \\
& \text { for } s=0.05 \text { : }
\end{aligned}
$$

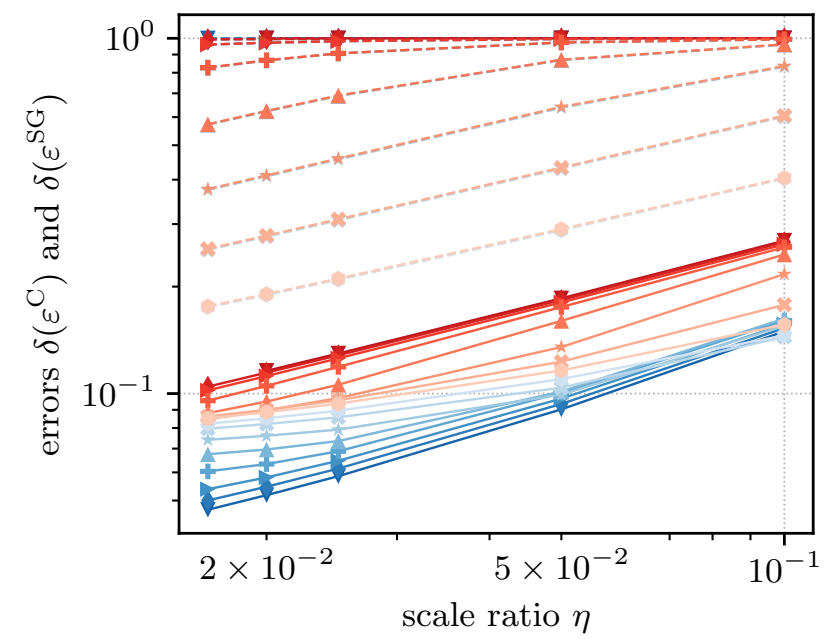

(b)

Figure 13: Approximation error, in terms of displacement field (left) and strain field (right), as a function of the scale ratio $\eta$, for the first-gradient model (dotted lines) and for the proposed strain-gradient model (solid lines). The two cases of microstructure relative position are represented: $s=0$ (red lines) and $s=0.1$ (blue lines)

with the error of the first-gradient model $\delta\left(\underline{\boldsymbol{u}}^{\mathrm{C}}\right)$. The effects of $\rho$ on the accuracy of the models will be further explored in the following section.

Similar observations can be made on Figure $12 \mathrm{~b}$ about the errors $\delta\left(\varepsilon^{\mathrm{SG}}\right)$ and $\delta\left(\varepsilon^{\mathrm{C}}\right)$ relative to the strain field. The gap between these two accuracy measures is less pronounced and the maximum of $\delta\left(\varepsilon^{\mathrm{SG}}\right)$ is now reached for $s=0.20$.

\subsection{Influence of the scale separation}

This second set of comparisons is focused on the influence of the scale separation and the junction thinness on the accuracy of the proposed strain-gradient model. A wide range of scale ratios is thus explored, $\eta=\frac{1}{N}$ for $N \in\{10 ; 20 ; 40 ; 50 ; 60\}$ as well as 8 values of the junction thinness $\rho$ ranging from $10^{-1}$ to $10^{-4}$. For the microstructure position, only two cases are considered in order to limit the number of investigated $(\rho, \eta, s)$ triplets. The first value $s=0.00$ is retained as it has been identified as the most favorable case in terms of accuracy of $\underline{\boldsymbol{u}}^{\mathrm{SG}}$ and $\varepsilon^{\mathrm{SG}}$ while the second value $s=0.05$ represents the case where the microstructure position is unfavorable. This second value is a compromise as it corresponds to a rather unfavorable case for both $\underline{\boldsymbol{u}}^{\mathrm{SG}}$ and $\boldsymbol{\varepsilon}^{\mathrm{SG}}$.

Trends when $\eta \rightarrow 0$. In Figure 13 the $L_{2}$ errors are plotted as functions of the scale ratio $\eta$ for both the first-gradient approximate solution and the strain-gradient one. The results for $s=0.00$ are shown in shades of blue and those for $s=0.05$ are shown in shades of red.

The displacement errors plotted on Figure 13a reveal a significant gap between the first-gradient 
and the strain-gradient model. Indeed, in most cases, the difference is larger than one decade and may reach two decades for some of the investigated parameters.

More precisely, when $\eta \rightarrow 0$ with rather thick junctions $\left(\rho \geq 3 \times 10^{-2}\right)$, we observe a steady decrease of the error of the first-gradient model $\delta\left(\underline{\boldsymbol{u}}^{\mathrm{C}}\right)$. The rate of this decrease is $\eta^{1}$ which corresponds to a classical result for a fixed $\rho$ (Jikov et al., 1994). When junctions become thinner, the decrease of $\delta\left(\underline{u}^{\mathrm{C}}\right)$ is delayed and becomes visible only if $\eta$ is sufficiently low revealing the influence of floppy modes on the homogenized response.

Regarding the strain-gradient model, for $s=0.05$ the error $\delta\left(\underline{\boldsymbol{u}}^{\mathrm{SG}}\right)$ decreases steadily when $\eta \rightarrow 0$ as well. The rate of decrease is similar to the one observed for the first-gradient model but with an amplitude one decade lower. In this case, thinner junctions lead to slightly higher values of $\delta\left(\underline{\boldsymbol{u}}^{\mathrm{SG}}\right)$ but this does not change the rate. For $s=0.00$ as well, $\delta\left(\underline{\boldsymbol{u}}^{\mathrm{SG}}\right)$ decreases almost steadily when $\eta \rightarrow 0$ and the rate is also close to $\eta^{1}$. Recall that the clamped boundary conditions of this benchmark problem create boundary layers and fall out assumptions made in (Smyshlyaev and Cherednichenko, 2000) for deriving a higher-order convergence result. Furthermore, there is a slight deviation from this trend: a small increase occurs when both $\eta$ and $\rho$ are small and $s=0.00$. Indeed, a deterioration of the full-scale solution probably occurs when $\rho \rightarrow 0$ and $\eta \rightarrow 0$, considering the size of the mesh (more than 1 million nodes), the significant contrast between the smallest elements which are inside the junctions and the largest elements in the triangles and the fact that the structure is loaded in a floppy mode. This deterioration of the reference solution is perceptible only in the case of the strain-gradient approximate solution and for $s=0.00$ because in this case the error is quite small $\left(\leq 5 \times 10^{-4}\right)$.

Focusing on the strain field, Figure 13b shows again the difference of accuracy between the two models. Similar trends can be noted for the two errors $\delta\left(\varepsilon^{\mathrm{C}}\right)$ and $\delta\left(\varepsilon^{\mathrm{SG}}\right)$ even though they are less clear than for the displacement errors. For the first-gradient model with thick junctions, the strain error $\delta\left(\varepsilon^{\mathrm{C}}\right)$ steadily decreases at a classical rate of $\eta^{\frac{1}{2}}$ (Jikov et al., 1994). The junction thinness has the same effect on the first-gradient error $\delta\left(\varepsilon^{\mathrm{C}}\right)$ and delays the emergence of the asymptotic trend. Steady decreases are also observed for the strain error resulting from the strain-gradient model $\delta\left(\varepsilon^{\mathrm{SG}}\right)$. However the rate of decrease is less clear. For a thin junction, it is close to $\eta^{\frac{1}{2}}$.

Trends when $\rho \rightarrow 0$. Now, our attention is turned to the effect of the junction thinness $\rho$. The errors are plotted in Figure 14 as functions of this parameter for both models. The same organization is used as for Figure 13.

When considering the first-gradient model, Figure 14a shows that the displacement error $\delta\left(\underline{\boldsymbol{u}}^{\mathrm{C}}\right)$ increases significantly as $\rho$ decreases until reaching a rather high plateau $\left(\delta\left(\underline{\boldsymbol{u}}^{\mathrm{C}}\right) \approx 1 \times 10^{-1}\right)$ beyond a $\rho$-threshold that depends on $\eta$.

In contrast, with the proposed strain-gradient model, the displacement error $\delta\left(\underline{\boldsymbol{u}}^{\mathrm{SG}}\right)$ varies only slightly when $\rho \rightarrow 0$. The junction thinness has less influence on $\delta\left(\underline{\boldsymbol{u}}^{\mathrm{SG}}\right)$ than the two other parameters, 


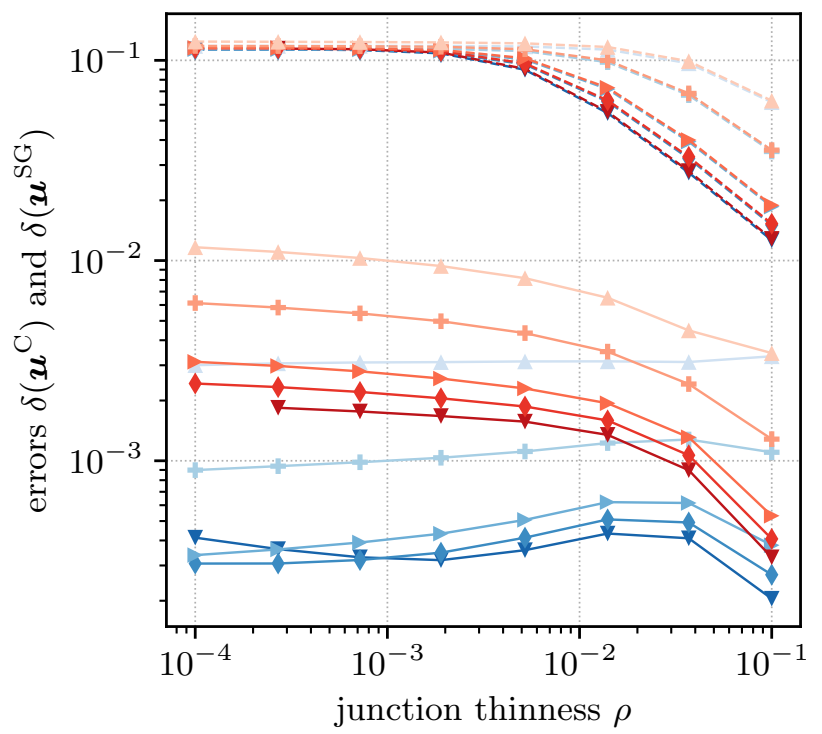

(a)

for $s=0.00$ :

for $s=0.05$ :

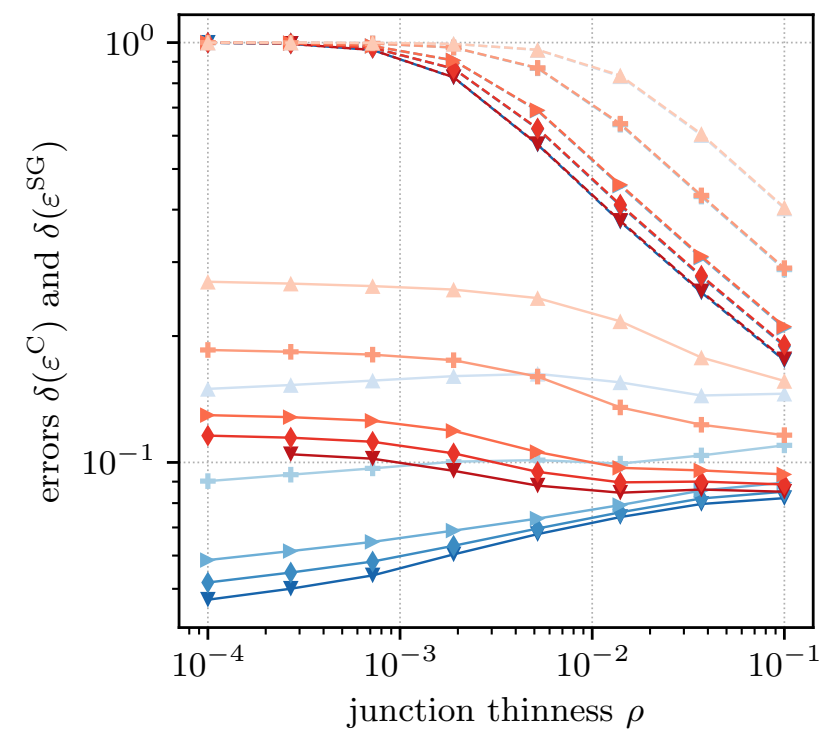

(b)

Figure 14: Approximation error, in terms of displacement field (left) and strain field (right), as a function of the junction thinness $\rho$ for both the first-gradient model reconstruction (dotted lines) and the proposed one (solid lines)

$\eta$ and $s$. Remarkably, for $s=0$, the error estimate is improved when junctions become thin whereas for $s=0.05$ the error increases moderately and remains limited for thin junctions. Focusing on the strain field, Figure 14b shows similar patterns for the strain errors $\delta\left(\varepsilon^{\mathrm{C}}\right)$ and $\delta\left(\varepsilon^{\mathrm{SG}}\right)$.

This comparison campaign shows that, for this metamaterial, the accuracy of the first-gradient model deteriorates quickly when $\rho \rightarrow 0$, even with a pronounced scale separation $(\eta=1 / 60)$. It also reveals that the proposed strain-gradient model is robust with respect to the junction thinness. Indeed, this model gives an approximate solution that is much more accurate than the one obtained with the first-gradient model whatever the junction thinness is.

\section{Conclusion}

In this paper, an architectured metamaterial called "pantographic material" made of polygons connected by compliant junctions was investigated. Contrary to architectured materials where junctions are assumed to be perfect and which can thus be described by a discrete modeling, the structure we consider is made of a single and continuous linear elastic material. This enables simple fabrication, favoring thus a future experimental campaign. It also enables to study the effects of some modification of the internal geometry, favoring thus a future topology optimization, for instance for generating meta-materials featuring the strongest strain-gradient effects (Calisti et al., 2021). Contrary also to structures based on very slender elements, which can still be described by a discrete modeling, the structure we consider contains only very localized thin parts: the "junctions". It is then not subject to local buckling, a phenomenon which drastically reduces the practical use of structures based on slender 
bars. The counterpart is the need a tool for computing the higher-order contributions in the elastic energy when the structure cannot be described by a discrete modeling.

To that aim a homogenization scheme based on higher-order contributions from the two-scale asymptotic expansion was suggested. When applied to the pantographic material made of compliant junctions, two scalings of the first-gradient homogenized stiffnesses came out as function of the junction thinness $\rho$, stiff modes scaling as $\frac{1}{|\log \rho|}$ whereas floppy modes scaling as $\rho^{2}$. This motivates the introduction of higher order terms of the asymptotic expansion. Whereas the second-order homogenized tensor may not be positive in general, it appeared that keeping significant contributions from the gradient of the eigenstrains of the first-order homogenized tensor ensures the positivity and the well-posedness of the proposed homogenized model.

The predictions from the homogenization scheme were investigated in detail and validated against a full-scale numerical simulation. For a fixed junction thinness, convergence rates predicted by the classical homogenization theory were retrieved. However, when junctions are becoming thinner the convergence is delayed and the classical homogenization scheme yields irrelevant predictions. On the contrary, the strain-gradient homogenization scheme that we propose delivers very good error estimates for either thin or thick junctions for a boundary value problem with a clamped boundary, which is known to challenge higher-order homogenized models.

Furthermore, we have checked that the relative position of the unit-cell does not have a significant influence on the quality of the prediction. Our results suggest that, even when the junction thickness tends to zero, the influence of the relative position of the microstructure on the accuracy of the proposed strain-gradient type approximate solution remains limited. Consequently, for microstructures that lead to significant strain-gradient effects, it seems unnecessary to muffle the influence of the microstructure relative position with a costly operation of averaging over all its possible values in the definition of the strain-gradient model.

Our full-scale numerical simulation has shown the limitation of such numerical simulations for studying the behavior of microstructured materials when they lead to strain-gradient effective behaviors. Indeed such behaviors appear only when the first-gradient stiffness tensor is almost degenerate which needs the presence of very compliant parts inside the unit-cell. These parts must be very finely meshed. As, on the other hand, homogenization takes sense only when the number of unit-cells is very large, we get a huge number of degrees of freedom and a poorly conditioned system. We are thus quickly limited both in the number of cells and in the thinness of junctions. This observation enforces the need of a trustful macroscopic model, that is of a safe upscaling method. We have suggested here a projection method and validated it on a benchmark problem. However we know that this method may fail (Jakabčin and Seppecher, 2020) for some microstructures which need to be described by an enriched continuum model. Future works should then establish sufficient hypotheses for ensuring that our projection method provides a good approximation of the global displacement of a microstructured 
material.

\section{Acknowledgment}

The authors acknowledge the support of the French Agence Nationale de la Recherche (ANR), under grant ANR-17-CE08-0039 (project ArchiMatHOS). This work was also partially funded by CNRS/IRP Coss\&Vita between Fédération Francilienne de Mécanique (F2M, CNRS FR2609) and $\mathrm{M} \& \mathrm{MoCS}$

\section{References}

M. Camar-Eddine, P. Seppecher, Determination of the Closure of the Set of Elasticity Functionals, Archive for Rational Mechanics and Analysis 170 (2003) 211-245.

E. M. P. Cosserat, F. Cosserat, Théorie des corps déformables, A. Hermann et fils, Paris, 1909.

R. Mindlin, Micro-structure in linear elasticity, Archive for Rational Mechanics and Analysis 16 (1964) $51-78$.

M. Kadic, G. W. Milton, M. van Hecke, M. Wegener, 3D metamaterials, Nature Reviews Physics 1 (2019) 198-210.

V. V. Jikov, S. M. Kozlov, O. A. Oleinik, Homogenization of Differential Operators and Integral Functionals, Springer Berlin Heidelberg, Berlin, Heidelberg, 1994.

M. P. Bendsøe, O. Sigmund, Topology Optimization, Springer Berlin Heidelberg, Berlin, Heidelberg, 2004.

S. Amstutz, S. M. Giusti, A. A. Novotny, E. A. de Souza Neto, Topological derivative for multiscale linear elasticity models applied to the synthesis of microstructures, International Journal for Numerical Methods in Engineering 84 (2010) 733-756.

F. Wang, O. Sigmund, J. Jensen, Design of materials with prescribed nonlinear properties, Journal of the Mechanics and Physics of Solids 69 (2014) 156-174.

B. Zhu, M. Skouras, D. Chen, W. Matusik, Two-scale topology optimization with microstructures, ACM Transactions on Graphics 36 (2017).

C. R. Thomsen, F. Wang, O. Sigmund, Buckling strength topology optimization of 2D periodic materials based on linearized bifurcation analysis, Computer Methods in Applied Mechanics and Engineering 339 (2018) 115-136.

G. Rosi, N. Auffray, Anisotropic and dispersive wave propagation within strain-gradient framework, Wave Motion 63 (2016) 120-134. 
E. H. Lee, W. H. Yang, On Waves in Composite Materials with Periodic Structure, SIAM Journal on Applied Mathematics 25 (1973) 492-499.

J. R. Willis, Polarization approach to the scattering of elastic waves-I. Scattering by a single inclusion, Journal of the Mechanics and Physics of Solids 28 (1980) 287-305.

J.-L. Auriault, G. Bonnet, Dynamique des composites élastiques périodiques., Arch. Mech. 37 (1985) 269-284.

H. Nassar, Q.-C. He, N. Auffray, Willis elastodynamic homogenization theory revisited for periodic media, Journal of the Mechanics and Physics of Solids 77 (2015) 158-178.

J. Vondřejc, E. Rohan, J. Heczko, Shape optimization of phononic band gap structures using the homogenization approach, International Journal of Solids and Structures 113-114 (2017) 147-168.

F. Santosa, W. W. Symes, A Dispersive Effective Medium for Wave Propagation in Periodic Composites, SIAM Journal on Applied Mathematics 51 (1991) 984-1005.

G. Allaire, M. Briane, M. Vanninathan, A comparison between two-scale asymptotic expansions and Bloch wave expansions for the homogenization of periodic structures, SeMA Journal 73 (2016) $237-259$.

M. Bonnet, R. Cornaggia, Higher order topological derivatives for three-dimensional anisotropic elasticity, ESAIM: Mathematical Modelling and Numerical Analysis 51 (2017) 2069-2092.

G. Allaire, T. Yamada, Optimization of dispersive coefficients in the homogenization of the wave equation in periodic structures, Numerische Mathematik 140 (2018) 265-326.

M. Bonnet, R. Cornaggia, B. B. Guzina, Microstructural Topological Sensitivities of the Second-Order Macroscopic Model for Waves in Periodic Media, SIAM Journal on Applied Mathematics 78 (2018) 2057-2082.

R. Cornaggia, C. Bellis, Tuning effective dynamical properties of periodic media by FFT-accelerated topological optimization, International Journal for Numerical Methods in Engineering 121 (2020) 3178-3205.

P. Germain, The Method of Virtual Power in Continuum Mechanics. Part 2: Microstructure, SIAM Journal on Applied Mathematics 25 (1973) 556-575.

M. Gologanu, J.-B. Leblond, G. Perrin, J. Devaux, Recent Extensions of Gurson's Model for Porous Ductile Metals, in: Continuum Micromechanics, Springer Vienna, Vienna, 1997, pp. 61-130. 
V. G. Kouznetsova, M. G. Geers, W. A. M. Brekelmans, Multi-scale constitutive modelling of heterogeneous materials with a gradient-enhanced computational homogenization scheme, International Journal for Numerical Methods in Engineering 54 (2002) 1235-1260.

N. Auffray, R. Bouchet, Y. Bréchet, Strain gradient elastic homogenization of bidimensional cellular media, International Journal of Solids and Structures 47 (2010) 1698-1710.

V. Monchiet, N. Auffray, J. Yvonnet, Strain-gradient homogenization: A bridge between the asymptotic expansion and quadratic boundary condition methods, Mechanics of Materials 143 (2020) 103309.

N. S. Bakhvalov, G. P. Panasenko, Homogenization: Averaging Processes in Periodic Media, Kluwer Academic Publishers, Dordrecht-Boston-London, 1989.

B. Gambin, E. Kröner, Higher-Order Terms in the Homogenized Stress-Strain Relation of Periodic Elastic Media, physica status solidi (b) 151 (1989) 513-519.

N. Triantafyllidis, S. Bardenhagen, The influence of scale size on the stability of periodic solids and the role of associated higher order gradient continuum models, Journal of the Mechanics and Physics of Solids 44 (1996) 1891-1928.

C. Boutin, Microstructural effects in elastic composites, International Journal of Solids and Structures 33 (1996) 1023-1051.

V. P. Smyshlyaev, K. D. Cherednichenko, On rigorous derivation of strain gradient effects in the overall behaviour of periodic heterogeneous media, Journal of the Mechanics and Physics of Solids 48 (2000) $1325-1357$.

R. H. Peerlings, N. A. Fleck, Numerical analysis of strain gradient effects in periodic media, Journal de Physique IV 11 (2001) Pr5-153-Pr5-160.

M. M. Ameen, R. H. Peerlings, M. G. Geers, A quantitative assessment of the scale separation limits of classical and higher-order asymptotic homogenization, European Journal of Mechanics, A/Solids 71 (2018) 89-100.

J.-J. Alibert, P. Seppecher, F. Dell'Isola, Truss Modular Beams with Deformation Energy Depending on Higher Displacement Gradients, Mathematics and Mechanics of Solids 8 (2003) 51-73.

F. Dell'Isola, I. Giorgio, M. Pawlikowski, N. L. Rizzi, Large deformations of planar extensible beams and pantographic lattices: heuristic homogenization, experimental and numerical examples of equilibrium, Proceedings of the Royal Society A: Mathematical, Physical and Engineering Sciences 472 (2016) 20150790 . 
F. Dell'Isola, P. Seppecher, M. Spagnuolo, E. Barchiesi, F. Hild, T. Lekszycki, I. Giorgio, L. Placidi, U. Andreaus, M. Cuomo, S. R. Eugster, A. Pfaff, K. Hoschke, R. Langkemper, E. Turco, R. Sarikaya, A. Misra, M. De Angelo, F. D'Annibale, A. Bouterf, X. Pinelli, A. Misra, B. Desmorat, M. Pawlikowski, C. Dupuy, D. Scerrato, P. Peyre, M. Laudato, L. Manzari, P. Göransson, C. Hesch, S. Hesch, P. Franciosi, J. Dirrenberger, F. Maurin, Z. Vangelatos, C. Grigoropoulos, V. Melissinaki, M. Farsari, W. Muller, B. E. Abali, C. Liebold, G. Ganzosch, P. Harrison, R. Drobnicki, L. Igumnov, F. Alzahrani, T. Hayat, Advances in pantographic structures: design, manufacturing, models, experiments and image analyses, Continuum Mechanics and Thermodynamics 31 (2019) 1231-1282.

C. Pideri, P. Seppecher, A second gradient material resulting from the homogenization of an heterogeneous linear elastic medium, Continuum Mechanics and Thermodynamics 9 (1997) 241-257.

H. Abdoul-Anziz, P. Seppecher, Homogenization of periodic graph-based elastic structures, Journal de l’École polytechnique — Mathématiques 5 (2018a) 259-288.

H. Abdoul-Anziz, P. Seppecher, Strain gradient and generalized continua obtained by homogenizing frame lattices, Mathematics and Mechanics of Complex Systems 6 (2018b) 213-250. Publisher: Mathematical Sciences Publishers.

H. Abdoul-Anziz, L. Jakabčin, P. Seppecher, Homogenization of an elastic material reinforced by very strong fibres arranged along a periodic lattice, Proceedings of the Royal Society A: Mathematical, Physical and Engineering Sciences 477 (2021) 20200620.

L. Jakabčin, P. Seppecher, On periodic homogenization of highly contrasted elastic structures, Journal of the Mechanics and Physics of Solids 144 (2020).

H. A. Anziz, Homogénéisation de composites élastiques périodiques à fort contraste : Conception de métamatériaux de second gradient, Ph.D. thesis, 2018.

B. Durand, A. Lebée, HO_homog python package: Pre-release, https://zenodo.org/record/ 4067101, 2020.

A. Logg, G. N. Wells, J. Hake, DOLFIN: a C++/Python Finite Element Library, in: A. Logg, K.-A. Mardal, G. N. Wells (Eds.), Automated Solution of Differential Equations by the Finite Element Method, Volume 84 of Lecture Notes in Computational Science and Engineering, Springer, 2012. Section: 10 .

C. Geuzaine, J.-F. Remacle, Gmsh: A 3-D finite element mesh generator with built-in pre- and post-processing facilities, Int. J. Numer. Meth. Engng 79 (2009) 1309-1331.

A. Flamant, Sur la répartition des pressions dans un solide rectangulaire chargé transversalement, Comptes Rendus de l'Académie des Sciences Paris 114 (1892) 1465-1468. 
J. H. Michell, On the Direct Determination of Stress in an Elastic Solid, with application to the Theory of Plates, Proceedings of the London Mathematical Society s1-31 (1899) 100-124.

M. S. Alnæs, J. Blechta, J. Hake, A. Johansson, B. Kehlet, A. Logg, C. Richardson, J. Ring, M. E. Rognes, G. N. Wells, The FEniCS Project Version 1.5, Archive of Numerical Software 3 (2015).

V. Calisti, A. Lebée, A. A. Novotny, J. Sokolowski, Sensitivity of the second order homogenized elasticity tensor to topological microstructural changes, submitted (2021). 